\title{
Closed-form stiffnesses of multi-bucket foundations for OWT including group effect correction factors*
}

\author{
J.D.R. Bordón ${ }^{\dagger 1}$, J.J. Aznárez ${ }^{1}$, L.A. Padrón ${ }^{1}$, O. Maeso ${ }^{1}$, and S. Bhattacharya ${ }^{2}$ \\ ${ }^{1}$ Instituto Universitario SIANI, Universidad de Las Palmas de Gran Canaria, 35017 Las Palmas de Gran Canaria, Spain \\ ${ }^{2}$ Department of Civil and Environmental Engineering, University of Surrey, GU2 7XH Guildford, United Kingdom
}

\begin{abstract}
Offshore Wind Turbine (OWT) support structures need to satisfy different Limit States (LS) such as Ultimate LS (ULS), Serviceability LS, Fatigue LS and Accidental LS. Furthermore, depending on the turbine rated power and the chosen design (all current designs are soft-stiff), target natural frequency requirements must also be met. Most of these calculations require the knowledge of the stiffnesses of the foundation which, especially in the case of large turbines in intermediate waters (30 to 60 meters), might need to be configured using multiple foundation elements. For this reason, this paper studies, for a homogeneous elastic halfspace, the static stiffnesses of groups of polygonally arranged non-slender suction bucket foundations in soft soils modeled as rigid solid embedded foundations. A set of formulas for correcting the stiffnesses obtained from isolated foundation formulation are proposed. It is shown through the study of several multi-megawatt OWTs that, as expected, group effects becomes more relevant as spacing decreases. Also, group effects are sensitive mainly to shear modulus of soil, foundation shape ratio and diameter, and the number of foundations. The results obtained from the soil-structure system show that ignoring group effects may add significant errors to the estimation of OWT fundamental frequencies and leads to either overestimating or underestimating it by $5 \%$. This highlights the importance of adequately modeling the interaction between elements of closely-separated multi-bucket foundations in soft soils, when current guidelines specify the target fundamental frequency to be at least $10 \%$ away from operational $1 \mathrm{P}$ and blade passing frequencies $(2 \mathrm{P} / 3 \mathrm{P}$ frequencies).
\end{abstract}

Keywords: offshore wind turbines, seabed foundations, soil-structure interaction, group effects, fundamental frequency

\section{Introduction}

Offshore wind development is spreading around the world, including now Asia and the United States. So far, the installations have been located near the coast, in shallow waters, i.e. water depths up to approximately 30 meters, using mainly monopile and gravity-based foundations. However, the expansion of this technology brings the need of locating wind turbines in more difficult zones of larger depths. For instance, in China, some problems have arisen due to the presence of soft soils in the available sites, and the need of foundations that withstand severe extreme conditions (hurricanes). In order to increase the available surface for Offshore Wind Turbines (OWT), foundation solutions for intermediate water depths between 30 and 60 meters are being studied. One of the proposed solutions consists in using multiple foundations, buckets (suction buckets/caissons) or piles, connected to a partially submerged substructure (conventional jacket, twisted jacket, multipod or other special designs) which supports the wind turbine [1, 2], see Fig. 1. Examples of multiple foundation technologies are found in the Chinese development, where foundations based on eight inclined piles are being built, see Fig. 22(a). Likewise, there are recent installations based on jackets supported on three or four bucket foundations in Europe, see Fig. 2(b).

The size of fixed OWT has been growing continuously since the early 2000s. As the turbines increase in power, the blades get longer, the Rotor-Nacelle-Assemblies (RNA) get heavier, and longer towers are required. Larger turbines are also slower (operating $1 \mathrm{P}$ range is lower) and, as a result, target natural frequencies decrease. In many cases, these natural frequencies will be very close to the dominant wave frequencies, thus making dynamic design very important [1]. Given the vast number of design requirements, see e.g. [3], simplified design procedures have been proposed [4, 5], which allow obtaining reasonable designs at the initial stages. In such procedures, among

\footnotetext{
*Pre-print of the article published in Marine Structures, 65, 326-342, 2019 (https://doi.org/10.1016/j.marstruc.2019.01.008)
}

${ }^{\dagger}$ Corresponding author: jacobdavid.rodriguezbordon@ulpgc.es 


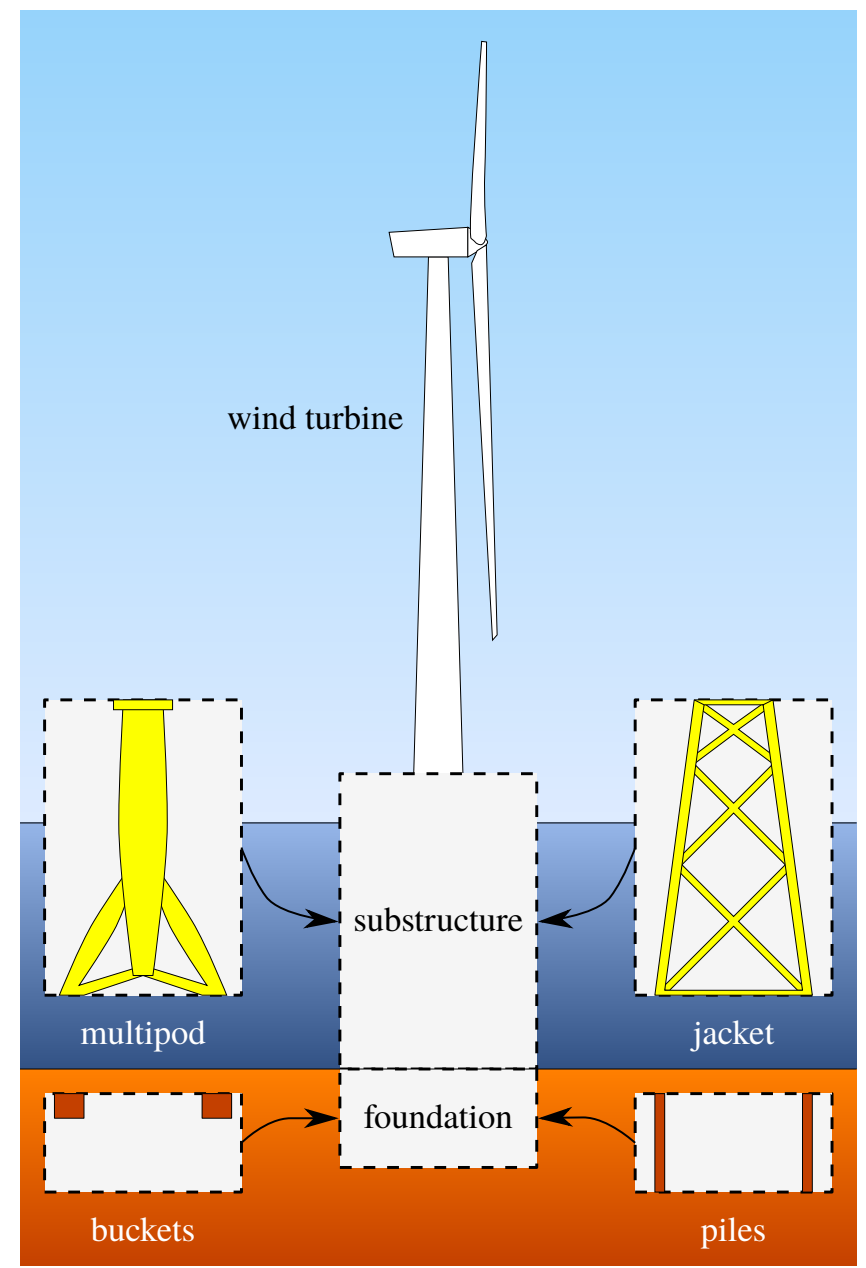

Figure 1: OWT foundation solutions for intermediate waters (30 to 60 meters) 


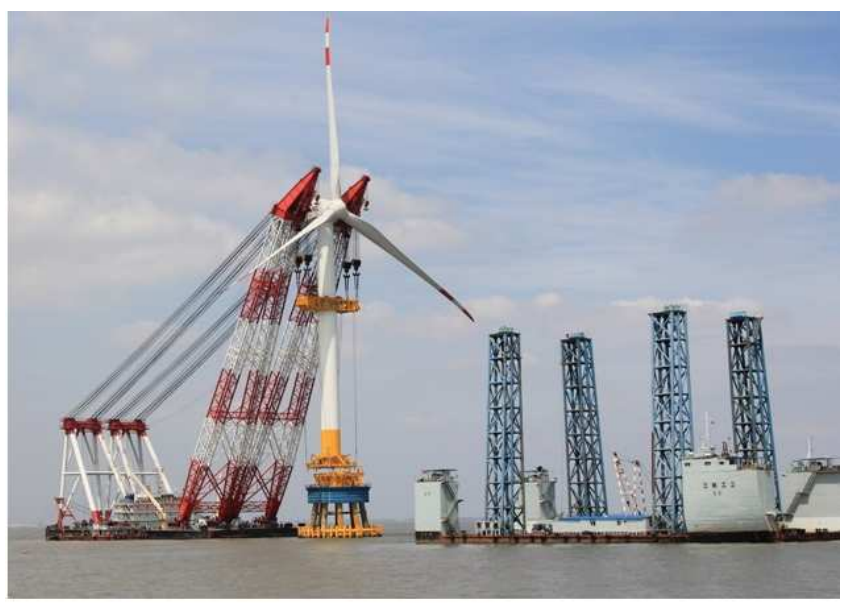

(a)

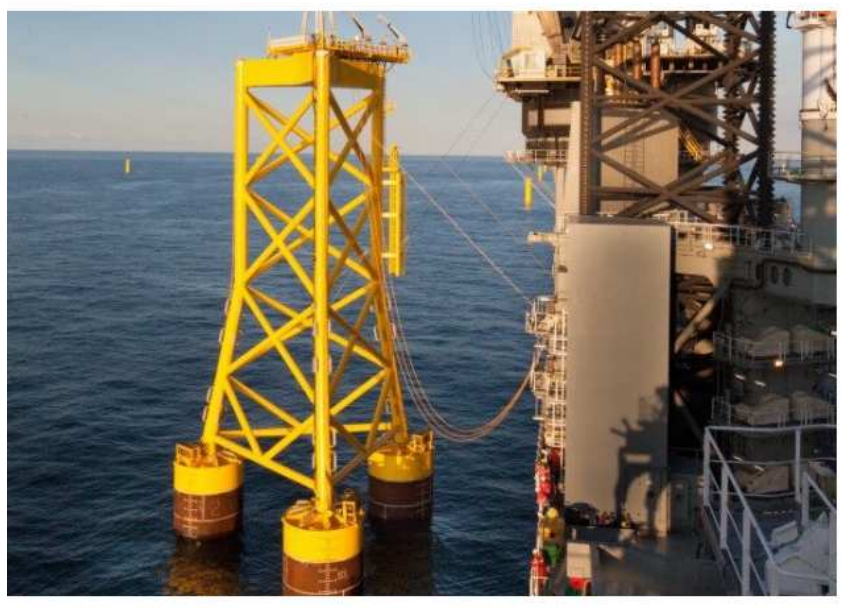

(b)

Figure 2: Multiple foundation examples: (a) Installation of a turbine on a eight inclined piles group, (b) Installation of a jacket supported on three suction bucket foundations (Vattenfall Project) 

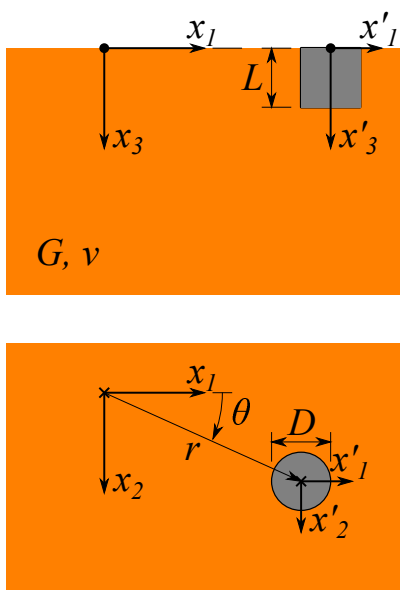

Figure 3: Isolated foundation
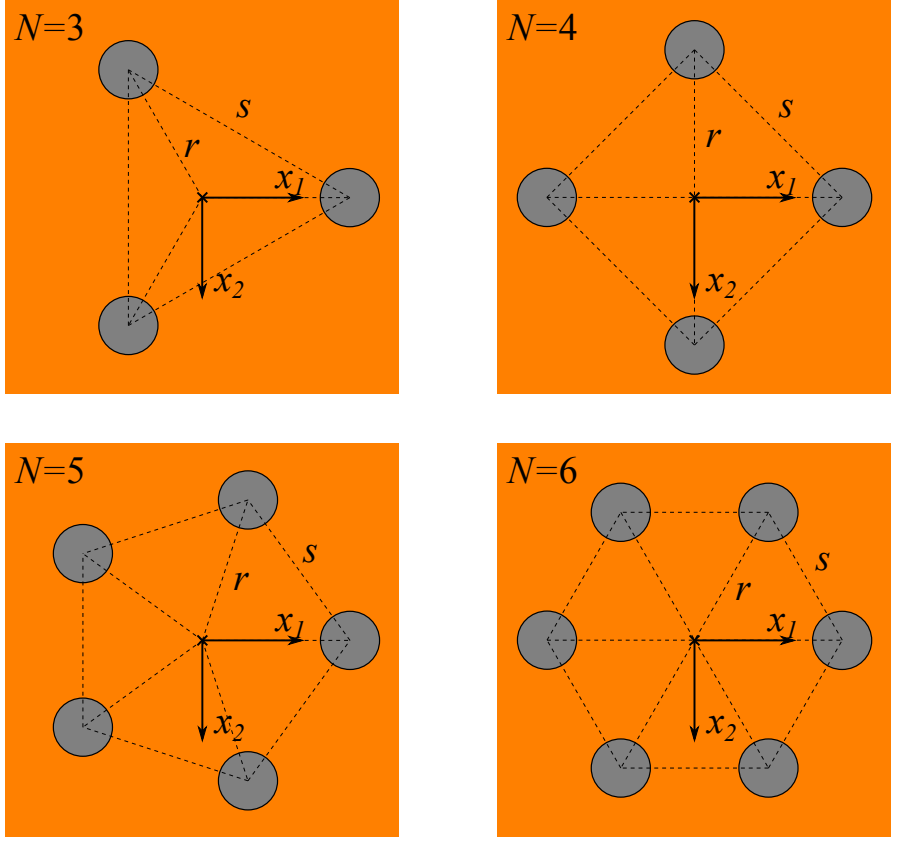

Figure 4: Layout of polygonally arranged groups of foundations

other aspects, foundation stiffnesses are used to assess Serviceability Limit State (SLS), Fatigue Limit State (FLS) and target natural frequency requirements. Because of the low value of the fundamental frequencies of OWTs $(0.2$ to $0.5 \mathrm{~Hz})$, the use of static stiffness matrices is usually accurate enough for taking into account soil-structure interaction when estimating their fundamental frequency [6, 7].

In this context, the main object of the present paper is to provide insight into the way in which the interaction between the different elements of an OWT multi-bucket foundation modify the global stiffness of this type of foundations. More precisely, the aim is twofold: (i) to offer a practical way of incorporating group effects into the computation of the stiffness of multi-bucket foundations by presenting a set of closed-form correction factors to the common simplified stiffness matrix built from the already known stiffnesses of individual isolated buckets; and (ii) to quantify the influence of considering these foundation group effects when using the resulting stiffnesses for computing the fundamental frequencies of Offshore Wind Turbines. To do this, the stiffnesses of the groups of foundations are computed using a three-dimensional boundary element model able to incorporate the geometry and material properties defining the problem, and capable of modeling the interactions between individual foundations. Polygonally arranged groups of buckets, embedded in a uniform elastic halfspace, are assumed. Four types of polygonal bases (tripod, tetrapod, pentapod, hexapod) are analyzed, and each individual foundation is taken as an rigid solid embedded cylindrical foundation, which is a reasonable simplifying assumption for modeling nonslender bucket foundations in soft soils [8, 9, 10].

The rest of the paper is organized as follows. Section 2 describes the problem at hand. Sections 3 and 4 describe respectively the rigorous methodology based on a boundary element model and the simplified (without group effect) methodology for the calculation of stiffnesses. Section 5 studies the group effects in detail, and a set of correction factors for the simplified methodology is proposed. In Section 6, the proposed correction factors are used to assess the relevance of group effects on the determination of the first natural frequency of OWT founded on such types of foundations. Finally, the main conclusions of the paper are given in Section 7

\section{Problem statement}

The soil is considered as an isotropic homogeneous elastic halfspace ( $\left.x_{3} \geq 0\right)$ with shear modulus $G$ and Poisson's ratio $0 \leq v<0.5$. Foundations are considered as rigid solid embedded cylindrical foundations with diameter $D$, length (foundation depth) $L$, and within the range $0 \leq L / D \leq 1$, bonded to the soil. Fig. 3 depicts the layout of a given foundation.

Foundations are located at vertices of a regular $N$-sided polygon with $N=3,4,5,6$, see Fig. 4 The polygon has radius $r$, and side length $s=2 r \sin (\pi / N)$ (foundation spacing). All foundations are considered to be rigidly connected, which constitutes a reasonable assumption for OWT jackets.

For these layouts, the present work studies the formulation of a closed-form static stiffness matrix for the 


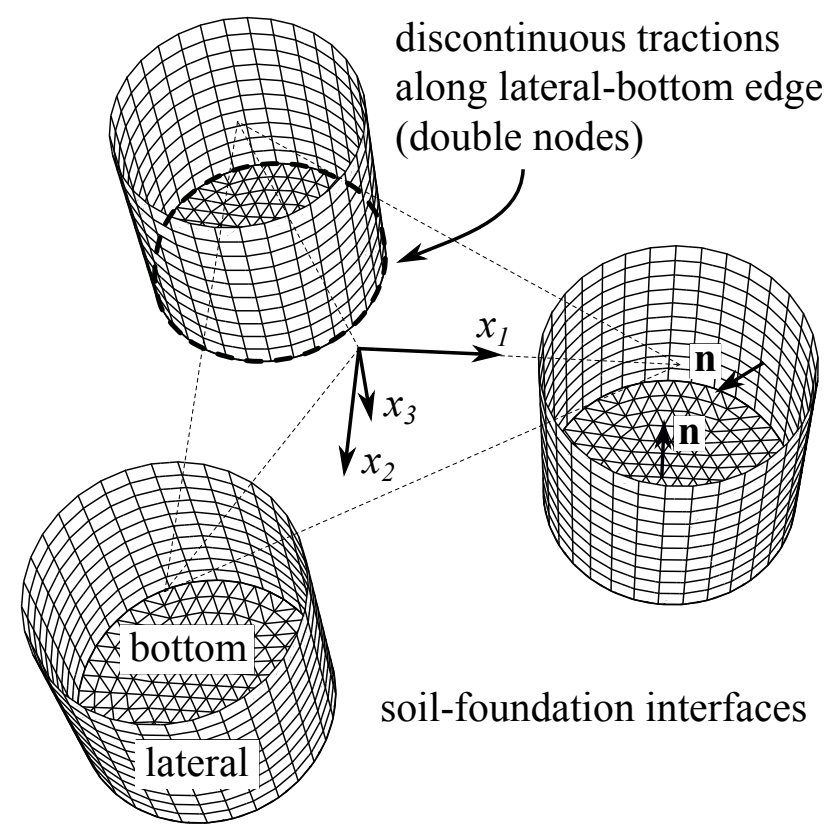

Figure 5: Example mesh used by the BEM numerical model $(N=3, L / D=1, s / D=2)$

system, where the six degrees of freedom stiffness matrix is reduced to the origin of coordinates (center of the polygon). In order to do so, a simplified stiffness matrix based on a rigid link of isolated foundations is considered first. Then, each element of this matrix is altered by correction factors due to group effects. Closed-form formulas of most of these correction factors are obtained by curve fitting of results from a three-dimensional continuum mechanics boundary element model.

\section{Rigorous numerical model}

A rigorous numerical model based on the Boundary Element Method (BEM) already developed by the authors [11, 12] is used to perform isolated and multiple foundation stiffness analyses. For the purpose of the present paper, the Mindlin's fundamental solution [13] has been included in it. The use of this fundamental solution avoid the discretization of the free-surface and only requires the discretization of the soil-foundation interface, providing a simple and accurate methodology.

The Singular Boundary Integral Equation (SBIE) used to build the linear system of equations reduces to:

$$
c_{l k}^{\mathrm{i}} u_{k}^{\mathrm{i}}+\int_{\Gamma_{\mathrm{sf}}} t_{l k}^{*} u_{k} \mathrm{~d} \Gamma=\int_{\Gamma_{\mathrm{sf}}} u_{l k}^{*} t_{k} \mathrm{~d} \Gamma
$$

where $c_{l k}^{\mathrm{i}}$ is the free-term [14], $u_{k}$ and $t_{k}=\sigma_{k j} n_{j}$ are respectively the displacement and traction vectors, $u_{l k}^{*}$ and $t_{l k}^{*}$ are the fundamental solutions in terms of respectively displacements and tractions. The superscript $\square^{\mathrm{i}}$ denotes variables related to the collocation (load) point, index $l=1,2,3$ is the load direction, index $k=1,2,3$ is the observation direction, and $\Gamma_{\mathrm{sf}}$ represents all soil-foundation boundaries. Discretization uses 9 node quadrilateral elements for foundation lateral and 6 node triangular elements for foundation bottom, as shown in Fig. 5 for a given configuration $(N=3, L / D=1, s / D=2)$. Unit normal vectors shown are oriented outward of the domain. Double nodes are used along the foundation bottom-lateral edge in order to allow discontinuous tractions there [12]. Boundary conditions for the stiffness calculation are given kinematically for all nodes as a rigid body motion:

- Vertical: $\mathbf{u}=(0,0,1)$.

- Horizontal: $\mathbf{u}=(1,0,0)$.

- Rocking: $\mathbf{u}=\left(0,-x_{3}, x_{2}\right)$.

- Torsional: $\mathbf{u}=\left(-x_{2}, x_{1}, 0\right)$.

Therefore, welded contact conditions are assumed for all foundation-soil interfaces. Given the symmetry properties of the foundation group with respect to the polygon center, results for horizontal and rocking modes are the same 
regardless of the use of any axis contained in the $x_{1}-x_{2}$ plane, except for small differences due to the discretization orientation with respect to the axis. Stiffnesses are given by the total soil reaction in terms of resultant forces and moments:

- Vertical: $K_{\mathrm{V}}^{\mathrm{gr}}=\int_{\Gamma_{\mathrm{sf}}} t_{3} \mathrm{~d} \Gamma$.

- Horizontal: $K_{\mathrm{H}}^{\mathrm{gr}}=\int_{\Gamma_{\mathrm{sf}}} t_{1} \mathrm{~d} \Gamma, K_{\mathrm{SR}}^{\mathrm{gr}}=\int_{\Gamma_{\mathrm{sf}}}\left(x_{3} t_{1}-x_{1} t_{3}\right) \mathrm{d} \Gamma$.

- Rocking: $K_{\mathrm{R}}^{\mathrm{gr}}=\int_{\Gamma_{\mathrm{sf}}}\left(x_{2} t_{3}-x_{3} t_{2}\right) \mathrm{d} \Gamma, K_{\mathrm{SR}}^{\mathrm{gr}}=-\int_{\Gamma_{\mathrm{sf}}} t_{2} \mathrm{~d} \Gamma$.

- Torsional: $K_{\mathrm{T}}^{\mathrm{gr}}=\int_{\Gamma_{\mathrm{sf}}}\left(x_{1} t_{2}-x_{2} t_{1}\right) \mathrm{d} \Gamma$.

which are obtained at the post-processing stage, once tractions $t_{k}$ from Eq. (1) are known after solving the resulting linear system of equations. It must be noticed that coupled sway-rocking stiffnesses obtained from both horizontal and rocking rigid body motions are the same, except for slight differences due to the discretization.

\section{Simplified stiffness matrix}

Without considering group effects, the stiffness matrix can be built in closed-form from previous results for isolated foundations. Only very few of these results have been obtained from purely analytical methods. They correspond to the very useful limiting case of a circular footing $(L=0)$ on uniform halfspace, where some simplifying foundationsoil contact assumptions are considered for some components. Remarkable works in this area are those of Bycroft [15] and Gerrard and Harrison [16] for horizontal loading, Spence [17] and Poulos and Davies [18] for vertical loading, Borowicka [19] for moment loading, and Reissner and Sagoci [20] for torsional loading. For embedded foundations, it is necessary to resort to semi-analytical or numerical methods, either the Finite Element Method or the Boundary Element Methods, whose results are usually in the form of tables, charts or formulas obtained from curve fitting. In this sense, it is important to highlight the line of work of Kausel [21, 22], Abascal [23], Domínguez [24] and Wolf [25, 26], who proposed different formulas and charts for static and dynamic stiffnesses of square and circular rigid embedded foundations. The reference work of Gazetas [27] collects and synthesizes results of dynamic stiffnesses in the form of formulas and charts for isolated embedded foundations of arbitrary basemat shapes (excluding annular shapes) for engineering practice. More recently, Doherty et al. [8, 9] obtained stiffnesses of embedded circular footings including flexible buckets for non-homogeneous elastic soils. Some of these results are suggested in several codes, such as the offshore standard DNV-OS-J101 [3] for design of OWT structures.

Despite these formulas are considered for engineering practice, they achieve relative errors as high as 20\% [28]. For the purposes of the present paper, it is necessary to obtain better approximations in order to correctly normalize and extract group effects correction factors. In this regard, the previously described boundary element model (see Section 3] for the isolated foundation is used for fitting formulas similar to those of Gazetas [27] but richer in parameters. The resulting formulas are presented in Appendix $\mathrm{A}$. Once the stiffnesses for isolated foundations are established, the six degrees of freedom load-displacement relationship at $x_{1}^{\prime}-x_{2}^{\prime}-x_{3}^{\prime}$ (see Fig. 3) can be written as $\mathbf{K}^{\mathrm{f}} \mathbf{u}^{\mathrm{f}}=\mathbf{f}^{\mathrm{f}}$ :

$$
\left[\begin{array}{cccccc}
K_{\mathrm{H}}^{\mathrm{f}} & 0 & 0 & 0 & K_{\mathrm{SR}}^{\mathrm{f}} & 0 \\
0 & K_{\mathrm{H}}^{\mathrm{f}} & 0 & -K_{\mathrm{SR}}^{\mathrm{f}} & 0 & 0 \\
0 & 0 & K_{\mathrm{V}}^{\mathrm{f}} & 0 & 0 & 0 \\
0 & -K_{\mathrm{SR}}^{\mathrm{f}} & 0 & K_{\mathrm{R}}^{\mathrm{f}} & 0 & 0 \\
K_{\mathrm{SR}}^{\mathrm{f}} & 0 & 0 & 0 & K_{\mathrm{R}}^{\mathrm{f}} & 0 \\
0 & 0 & 0 & 0 & 0 & K_{\mathrm{T}}^{\mathrm{f}}
\end{array}\right]\left\{\begin{array}{c}
u_{x_{1}^{\prime}}^{\mathrm{f}} \\
u_{x_{2}^{\prime}}^{\mathrm{f}} \\
u_{x_{3}^{\prime}}^{\mathrm{f}} \\
\theta_{x_{1}^{\prime}}^{\mathrm{f}} \\
\theta_{x_{2}^{\prime}}^{\mathrm{f}} \\
\theta_{x_{3}^{\prime}}^{\mathrm{f}}
\end{array}\right\}=\left\{\begin{array}{c}
F_{x_{1}^{\prime}}^{\mathrm{f}} \\
F_{x_{2}^{\prime}}^{\mathrm{f}} \\
F_{x_{3}^{\prime}}^{\mathrm{f}} \\
M_{x_{1}^{\prime}}^{\mathrm{f}} \\
M_{x_{2}^{\prime}}^{\mathrm{f}} \\
M_{x_{3}^{\prime}}^{\mathrm{f}}
\end{array}\right\}
$$

where the superscript $\square^{\mathrm{f}}$ is used to emphasize that these terms correspond to an isolated foundation. Since the foundation is axisymmetric, only five stiffnesses are present in the relationship between displacements/rotations and forces/moments: vertical $K_{\mathrm{V}}^{\mathrm{f}}$, horizontal (or lateral) $K_{\mathrm{H}}^{\mathrm{f}}$, rocking $K_{\mathrm{R}}^{\mathrm{f}}$, sway-rocking $K_{\mathrm{SR}}^{\mathrm{f}}\left(K_{\mathrm{SR}}^{\mathrm{f}}>0\right)$ and torsional $K_{\mathrm{T}}^{\mathrm{f}}$. Ignoring group effects, it is possible to obtain a simple closed-form stiffness matrix with respect to the center $x_{1}-x_{2}-x_{3}$ of a rigidly connected set of axisymmetric foundations located at the vertices of a regular polygon whose circumcircle radius is $r$, see Fig. 4. It is straightforward to obtain by inspection that the vertical, horizontal 
and sway-rocking stiffnesses of the foundation group $\left(\mathbf{K}^{\mathrm{grs}} \mathbf{u}^{\mathrm{grs}}=\mathbf{f}^{\mathrm{grs}}\right)$ as:

$$
\begin{aligned}
& K_{\mathrm{V}}^{\mathrm{grs}}=N K_{\mathrm{V}}^{\mathrm{f}} \\
& K_{\mathrm{H}}^{\mathrm{grs}}=N K_{\mathrm{H}}^{\mathrm{f}} \\
& K_{\mathrm{SR}}^{\mathrm{grs}}=N K_{\mathrm{SR}}^{\mathrm{f}}
\end{aligned}
$$

where $N \geq 3$ is the number of vertices. Moreover, considering that that the rocking stiffness $K_{\mathrm{R}}^{\text {grs }}$ of the foundation group depends on a rotation axis a contained in the $x_{1}-x_{2}$ plane and defined by $\mathbf{a}=(\cos \psi, \sin \psi, 0)$, the following equation is obtained:

$$
K_{\mathrm{R}}^{\mathrm{grs}}(\psi)=N K_{\mathrm{R}}^{\mathrm{f}}+\left[\sum_{k=1}^{k=N} \sin ^{2}(\psi+2 \pi(k-1) / N)\right] r^{2} K_{\mathrm{V}}^{\mathrm{f}}
$$

where summation turns out to be constant and equal to $N / 2$, i.e. the resulting foundation is also axisymmetric. Therefore, the rocking stiffness $K_{\mathrm{R}}^{\mathrm{grs}}$ is:

$$
K_{\mathrm{R}}^{\mathrm{grs}}=N\left(K_{\mathrm{R}}^{\mathrm{f}}+\frac{1}{2} r^{2} K_{\mathrm{V}}^{\mathrm{f}}\right)
$$

The torsional stiffness $K_{\mathrm{T}}^{\mathrm{gr}}$ of the foundation group is simply:

$$
K_{\mathrm{T}}^{\mathrm{grs}}=N\left(K_{\mathrm{T}}^{\mathrm{f}}+r^{2} K_{\mathrm{H}}^{\mathrm{f}}\right)
$$

Therefore, the stiffness matrix $\mathbf{K}^{\text {grs }}$ of the foundation group can be written as:

$$
\mathbf{K}^{\mathrm{grs}}=N\left[\begin{array}{cccccc}
K_{\mathrm{H}}^{\mathrm{f}} & 0 & 0 & 0 & K_{\mathrm{SR}}^{\mathrm{f}} & 0 \\
0 & K_{\mathrm{H}}^{\mathrm{f}} & 0 & -K_{\mathrm{SR}}^{\mathrm{f}} & 0 & 0 \\
0 & 0 & K_{\mathrm{V}}^{\mathrm{f}} & 0 & 0 & 0 \\
0 & -K_{\mathrm{SR}}^{\mathrm{f}} & 0 & K_{\mathrm{R}}^{\mathrm{f}}+r^{2} K_{\mathrm{V}}^{\mathrm{f}} / 2 & 0 & 0 \\
K_{\mathrm{SR}}^{\mathrm{f}} & 0 & 0 & 0 & K_{\mathrm{R}}^{\mathrm{f}}+r^{2} K_{\mathrm{V}}^{\mathrm{f}} / 2 & 0 \\
0 & 0 & 0 & 0 & 0 & K_{\mathrm{T}}^{\mathrm{f}}+r^{2} K_{\mathrm{H}}^{\mathrm{f}}
\end{array}\right]
$$

This stiffness matrix can also be obtained in a systematic way by using rigid links through a master-slave relationship [29] as:

$$
\mathbf{K}^{\mathrm{grs}}=\sum_{k=1}^{k=N}[\mathbf{T}(\psi, k, N)]^{T} \cdot \mathbf{K}^{\mathrm{f} k} \cdot \mathbf{T}(\psi, k, N)
$$

where $\mathbf{K}^{\mathrm{f} k}=\mathbf{K}^{\mathrm{f}}$ since all foundations are equal. The master-slave transformation matrix $\left(\mathbf{u}^{\mathrm{f} k}=\mathbf{T}(\psi, k, N) \cdot \mathbf{u}^{\mathrm{grs}}\right)$ is:

$$
\mathbf{T}(\psi, k, N)=\left[\begin{array}{cccccc}
1 & 0 & 0 & 0 & r_{3}(\psi, k, N) & -r_{2}(\psi, k, N) \\
0 & 1 & 0 & -r_{3}(\psi, k, N) & 0 & r_{1}(\psi, k, N) \\
0 & 0 & 1 & r_{2}(\psi, k, N) & -r_{1}(\psi, k, N) & 0 \\
0 & 0 & 0 & 1 & 0 & 0 \\
0 & 0 & 0 & 0 & 1 & 0 \\
0 & 0 & 0 & 0 & 0 & 1
\end{array}\right]
$$

where the master-slave distance vector components are:

$$
\begin{aligned}
& r_{1}(\psi, k, N)=r \cos (\psi+2 \pi(k-1) / N) \\
& r_{2}(\psi, k, N)=r \sin (\psi+2 \pi(k-1) / N) \\
& r_{3}(\psi, k, N)=0
\end{aligned}
$$




\section{Group effects study}

\subsection{Vertical stiffness}

The group effects under static vertical loading of a group of foundations is qualitatively very well known from a physical point of view, see e.g. [30, 31] for pile groups. In order to describe qualitatively the phenomena for this and all other stiffnesses, we simply consider a two foundation system, where a rigid body motion is given to one of the foundations while the other remain free (unloaded and unconstrained) in the soil mass. When a vertical rigid body motion is given to one of the foundations, the other free-standing foundation coherently moves vertically, horizontally and also tilts according to the soil displacement field (see Fig. 6). This means that there is a vertical-vertical, vertical-horizontal and vertical-rocking mutual interaction between foundations. As the present case is concerned, the vertical-horizontal and vertical-rocking mutual interactions cancel out from the group point of view due to the symmetry of the foundation layout. The vertical-vertical interaction produces a helping effect, thus group effects from the vertical stiffness point of view is an effective reduction of its value when compared to the simple addition obtained from matrix analysis in section 4 through Eq. (3).

Fig. 7 shows the obtained correction factor $\gamma_{\mathrm{V}}=K_{\mathrm{V}}^{\mathrm{gr}} / K_{\mathrm{V}}^{\mathrm{grs}}$ for the vertical stiffness using solid lines, where $K_{\mathrm{V}}^{\mathrm{gr}}$ is obtained using the BEM model and $K_{\mathrm{V}}^{\mathrm{grs}}$ is obtained from (Eq. (3)). As expected, the correction factor tends to 1 as $s / D \rightarrow \infty$, and it does this monotonically from a minimum value between $0.36(N=6, L / D=1)$ and 0.61 $(N=3, L / D=0)$ obtained when $s / D \rightarrow 1$. For a given spacing $s / D$, the correction factor is more severe as $N$ and/or $L / D$ increase, which is a reasonable behavior. Roughly speaking, the correction factor is approximately 0.7 for $s / D=3$. On the other hand, it is observed that it has a relatively small sensitivity to the Poisson's ratio, except for high $L / D$. The presented correction factors have been validated for the case $L / D=0$ using the methodology developed by Randolph and Wroth [32], showing practically identical results.

Given Boussinesq's solution, see e.g. [13], and the obtained results, it is reasonable to consider a rational function of the type $1 /(1+f(N, v, L / D) /(s / D))$ for approximating the correction factor. The following simple formula has been obtained via curve fitting:

$$
\gamma_{\mathrm{V}}=\frac{K_{\mathrm{V}}^{\mathrm{gr}}}{K_{\mathrm{V}}^{\mathrm{grs}}} \approx \frac{1}{1+0.11(1+1.68 N)\left(1+0.71(L / D)^{0.76}\right) /(s / D)}
$$

where the influence of the Poisson's ratio has been neglected. For the curve fitting, BEM results from the full combination of the following parameters is considered: $N=\{3,4,5,6\}, s / D=\{1.01,1.1,1.25,1.5,2,2.5,3,3.5$, $4,4.5,5,7.5,10,15,20,30,50,100\}, L / D=\{0,0.125,0.25,0.5,0.75,1\}, v=\{0,0.1,0.2,0.3,0.4,0.49\}$; which constitutes 2592 cases in total. The same cases are also considered for the curve fitting of the rest of correction factors. The average relative error $\left(\sum\left|\left(\gamma_{V}^{\text {formula }}-\gamma_{V}^{\text {reference }}\right) / \gamma_{V}^{\text {reference }}\right| / N_{\text {cases }}\right)$ of this formula is $1.6 \%$, while the maximum relative error reached $\left(\max \mid\left(\gamma_{V}^{\text {formula }}-\gamma_{V}^{\text {reference }}\right) / \gamma_{V}^{\text {reference }}\right)$ is $10.7 \%$. The goodness of this formula is shown in Fig. 7] using dashed lines.

\subsection{Horizontal stiffness}

The group effects under static horizontal loading of a group of foundations is qualitatively very similar to that of the vertical loading. By using again the two foundation example, it is possible to observe in Fig. 8 that the freestanding foundation coherently moves horizontally with the soil mass, but it also moves vertically and tilts due to the variation of the vertical displacement of the soil mass. Torsion is also present when the horizontal movement is not aligned with both foundations. For the polygonal layout, horizontal-torsion mutual interactions cancel out from the group point of view due to its symmetry. On the other hand, horizontal-rocking mutual interactions do not cancel out and group effects related to the sway-rocking stiffness is also present. This effect is studied in section 5.4. As it happened for the vertical stiffness, group effects on the horizontal stiffness produce an effective reduction of its value when compared to the simple matrix analysis shown in section 4 through Eq. (4).

Fig. 9 shows the obtained correction factor $\gamma_{\mathrm{H}}=K_{\mathrm{H}}^{\mathrm{gr}} / K_{\mathrm{H}}^{\mathrm{grs}}$ for the horizontal stiffness using solid lines, where $K_{\mathrm{H}}^{\mathrm{gr}}$ is obtained using the BEM model and $K_{\mathrm{H}}^{\mathrm{grs}}$ is obtained from (Eq. (4)). As in the vertical loading case, Poisson's ratio has small influence on the correction factor for horizontal stiffness. In this case, however, Poisson's ratio becomes more relevant for small $L / D$. It has been verified the good agreement between BEM and Wong and Luco [33] horizontal correction factor results for a two foundation system with $L / D=0$.

Given Cerruti's solution, see e.g. [13], and the obtained results, it is reasonable to consider again a rational function of the type $1 /(1+f(N, v, L / D) /(s / D))$ for approximating this correction factor. The following simple formula has been obtained via curve fitting of the results:

$$
\gamma_{\mathrm{H}}=\frac{K_{\mathrm{H}}^{\mathrm{gr}}}{K_{\mathrm{H}}^{\mathrm{grs}}} \approx \frac{1}{1+0.06(1+3.08 N)\left(1+1.2(L / D)^{0.53}\right) /(s / D)}
$$




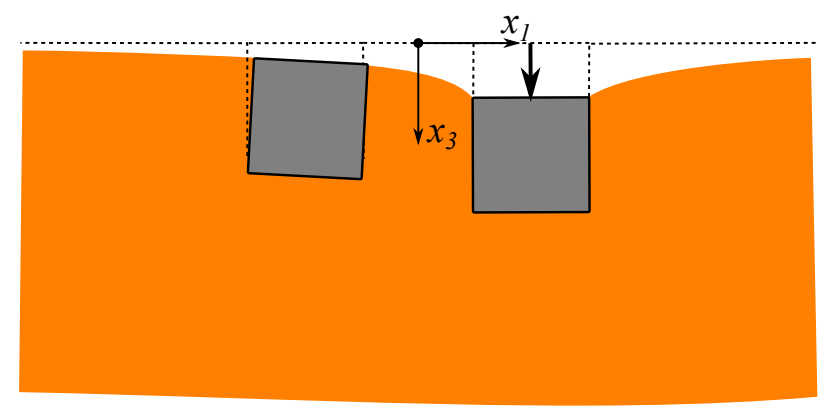

Figure 6: Illustration of the effect of a foundation vertical movement (right) over another free-standing foundation (left)
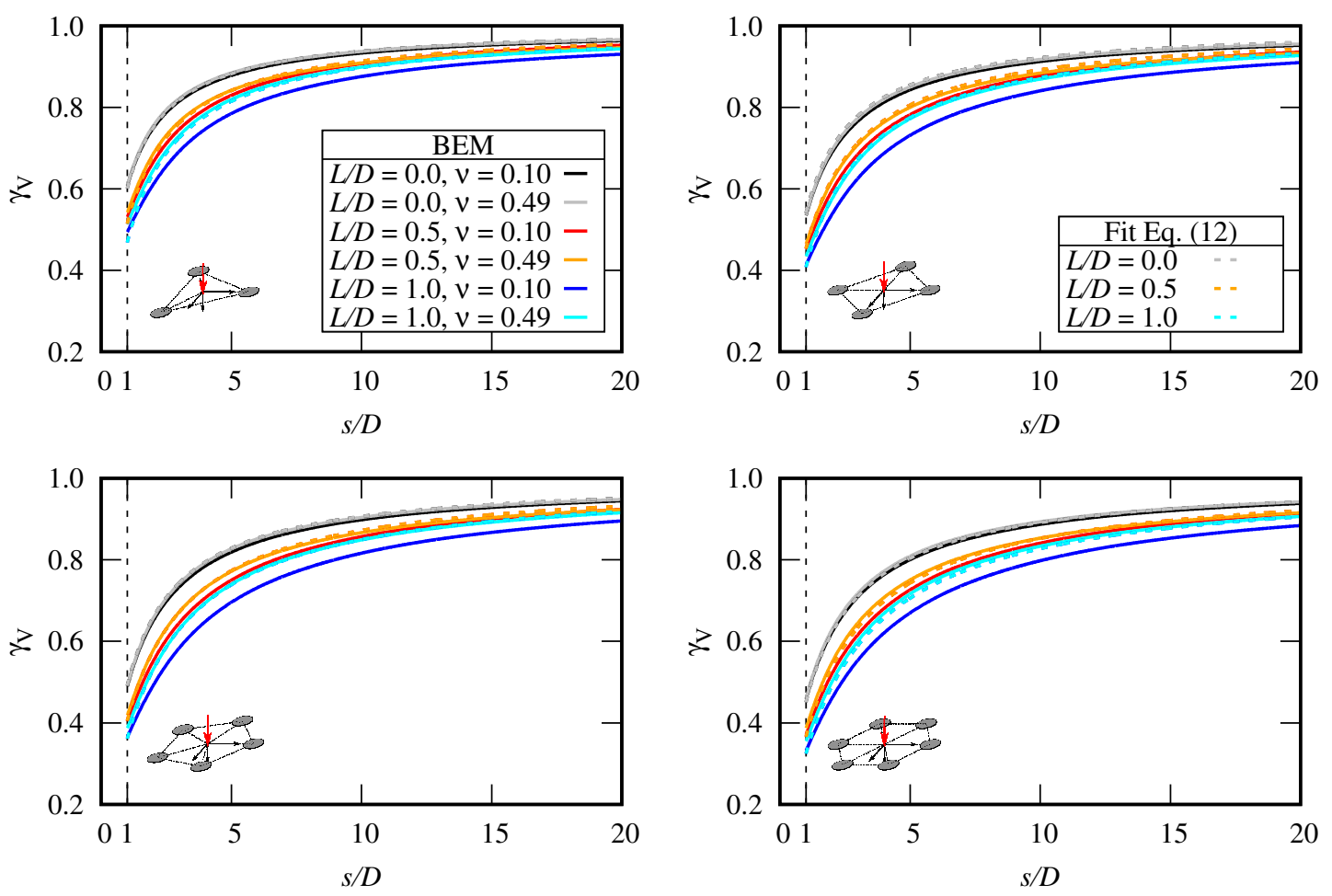

Figure 7: Correction factor $\gamma_{\mathrm{V}}=K_{\mathrm{V}}^{\mathrm{gr}} / K_{\mathrm{V}}^{\mathrm{grs}}$ for the vertical stiffness 


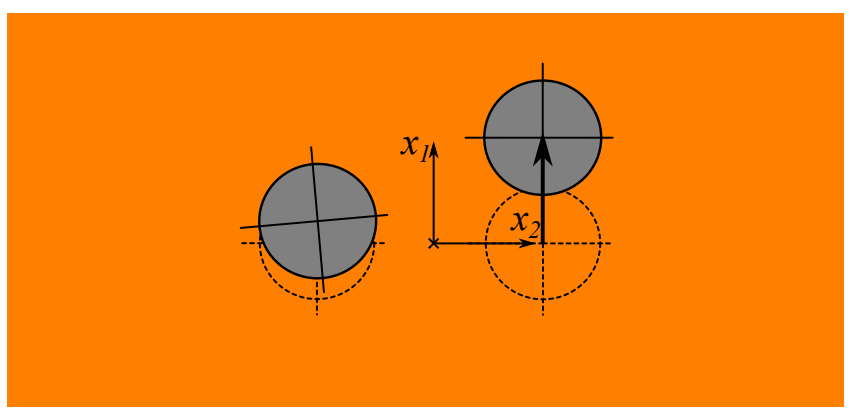

(a) Movement transverse to both foundations

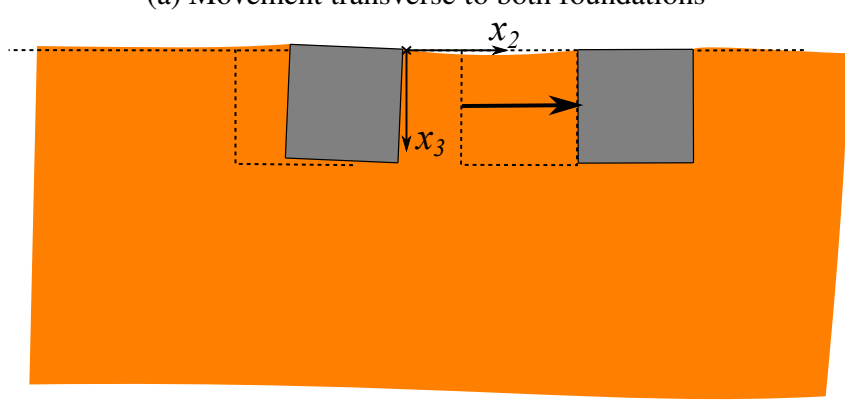

(b) Movement aligned with both foundations

Figure 8: Illustration of the effect of a foundation horizontal movement (right) over another free-standing foundation (left)
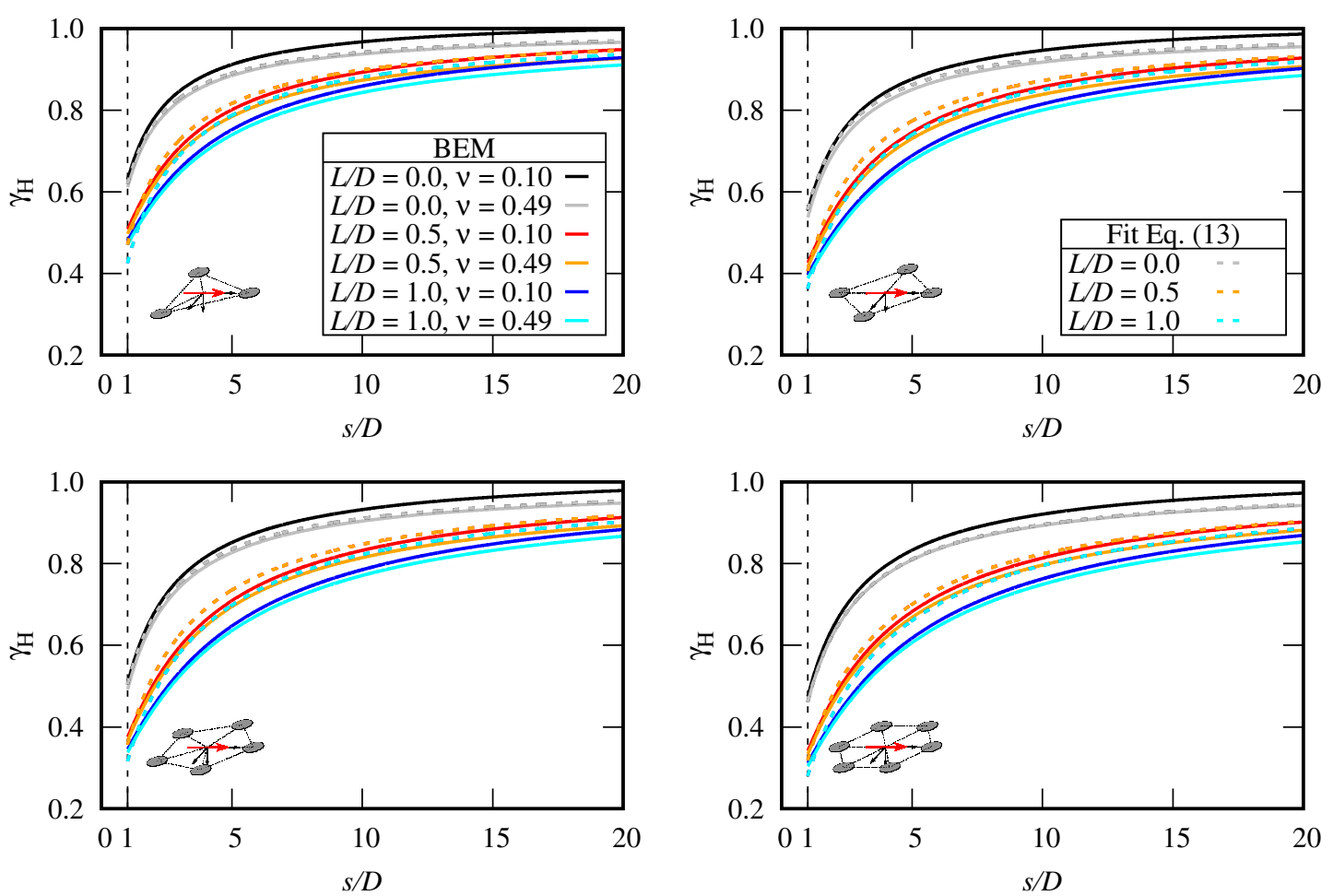

Figure 9: Correction factor $\gamma_{\mathrm{H}}=K_{\mathrm{H}}^{\mathrm{gr}} / K_{\mathrm{H}}^{\mathrm{grs}}$ for the horizontal stiffness 
where the influence of the Poisson's ratio has been neglected. The average relative error of this formula is $3.1 \%$, while the maximum relative error reached is $12 \%$. The goodness of the proposed formula is shown in Fig. 9 using dashed lines.

\subsection{Rocking stiffness}

The group effects under static rocking loading of a group of foundations are in some aspects different to vertical and horizontal loadings, although they share some aspects to the former. Again, a two foundation example is considered to offer a physical explanation to the results, see Figs. 10 and 11. While in the case of vertical and horizontal rigid body motions the translation of one foundation translates the other free-standing foundation in the same direction, i.e. there is a helping effect, in the case of rocking rigid body motion there are helping but also counteraction effects. The rotation of a foundation about its own axis produces a rotation in the same direction on the other foundation if it is located along the rotation axis, but it produces a rotation in the opposite direction if the free-standing foundation is located perpendicular to the rotation axis (see Fig. 10). The vertical displacement of a foundation due to its rotation about an axis passing through the center of the polygon produces counteracting effects if the free-standing foundation is located on the opposite rotation side (see Fig. 11a), but it produces helping effects if the other foundation is located on the same rotation side (see Fig. 11b).

Fig. 12 shows the obtained correction factor $\gamma_{\mathrm{R}}=K_{\mathrm{R}}^{\text {gr }} / K_{\mathrm{R}}^{\text {grs }}$ for the rocking stiffness using solid lines, where $K_{\mathrm{R}}^{\mathrm{gr}}$ is obtained using the BEM model and $K_{\mathrm{R}}^{\mathrm{grs}}$ is obtained from (Eq. (77). Due to the dominance of counteracting effects for large spacings, the correction factor approaches unity as $s / D \rightarrow \infty$ from correction factors greater than one. This behavior is completely the opposite to what happens to the correction factors of vertical and horizontal stiffnesses. Another opposite feature is the fact that the correction factor is not monotonous, which is due to the presence of helping as well as counteracting effects on foundations with respect to the rocking mode. A peak in the rocking stiffness of the group is observed for $s / D$ between 1 and 4 , being more noticeable for small $N$ and $L / D$. The group effects as $s / D \rightarrow 1$ is however similar to the vertical and horizontal correction factors, showing a stiffness decrease of the group but with a much faster stiffness change. There is sudden variations of the correction factor from $\gamma_{\mathrm{R}} \approx 0.7$ at $s / D=1$ to $\gamma_{\mathrm{R}} \approx 1$ at $s / D=3$. Poisson's ratio has greater influence on the correction factor than in vertical or horizontal correction factors, but it still remains of secondary importance.

As in previous cases, it is reasonable to consider again a rational function for approximating the correction factor, but in this case the denominator is enriched with a $(s / D)^{2}$ term, i.e. $1 /\left(1+f_{1}(N, v, L / D) /(s / D)+\right.$ $\left.f_{2}(N, v, L / D) /(s / D)^{2}\right)$. The following simple formula has been obtained via curve fitting of the results:

$$
\begin{aligned}
\gamma_{\mathrm{R}} & =\frac{K_{\mathrm{R}}^{\mathrm{gr}}}{K_{\mathrm{R}}^{\mathrm{grs}}} \approx \frac{1}{1+f_{1 \mathrm{R}} /(s / D)+f_{2 \mathrm{R}} /(s / D)^{2}} \\
f_{1 \mathrm{R}} & =-0.67(1-0.13 N)(1-0.53 v)\left(1+0.35(L / D)^{0.49}\right) \\
f_{2 \mathrm{R}} & =0.29(1-0.04 N)(1-0.12 v)(1+2.87(L / D))
\end{aligned}
$$

The average relative error of this formula is $1.2 \%$, while the maximum relative error reached is $6.7 \%$. The goodness of the proposed formula is shown in Fig. 9 using dashed lines.

\subsection{Coupled sway-rocking stiffness}

The group effect related to the coupled sway-rocking stiffness has an unexpected and very interesting behavior. While the other stiffnesses tend to the values obtained from isolated foundations as the spacing increases, the swayrocking stiffness tend to a different limiting value, as shown in Fig. 13 To the best knowledge of the authors, this type of behavior is not present in the literature. We have investigated this issue by analyzing the numerical results, and we have found a theoretical answer based on the use of point load solutions.

Fig. 13 shows the obtained correction factor $\gamma_{\mathrm{SR}}=K_{\mathrm{SR}}^{\mathrm{gr}} / K_{\mathrm{SR}}^{\mathrm{grr}}$ for the sway-rocking stiffness, where $K_{\mathrm{SR}}^{\mathrm{gr}}$ is obtained using the BEM model and $K_{\mathrm{SR}}^{\text {grs }}$ is obtained from (Eq. (5)). The resulting stiffnesses are greater than those obtained from isolated foundations when $s / D \rightarrow \infty$. By examining the numerical results regarding the horizontal mode, it is observed that despite the vertical resultant forces on each foundation vanish as $s / D \rightarrow \infty$, the product of these forces by their distance to the rotation axis of the foundation system tends to a finite value. This produces an additional moment, which added to their own moments (similar to those of an isolated foundation), gives the resulting increased stiffness. An analogous behavior is also observed for the rocking mode and the horizontal resultant, as expected from the stiffness matrix symmetry.

In order to evaluate this additional stiffness, we consider a convenient system of point loads at the interior of the halfspace using Mindlin's solution [13], which collapses into Cerruti's solution [34] when the load is applied at the free-surface. The use of point loads is justified since it is the behavior as $r \rightarrow \infty$ which is being studied. 


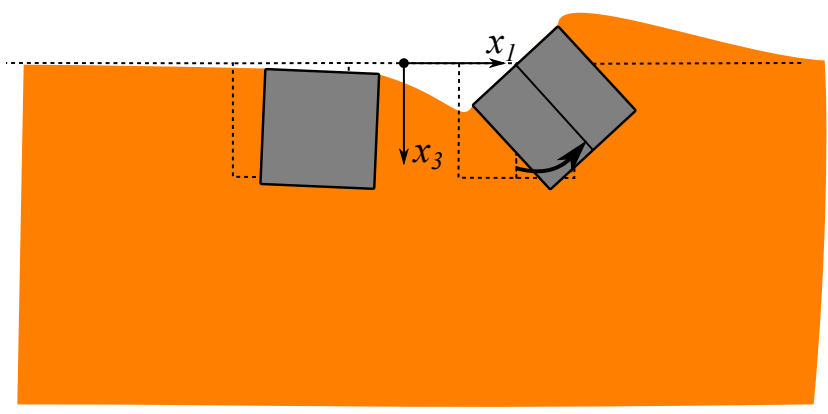

Figure 10: Illustration of the effect of a foundation rocking movement (right) over another free-standing foundation (left)

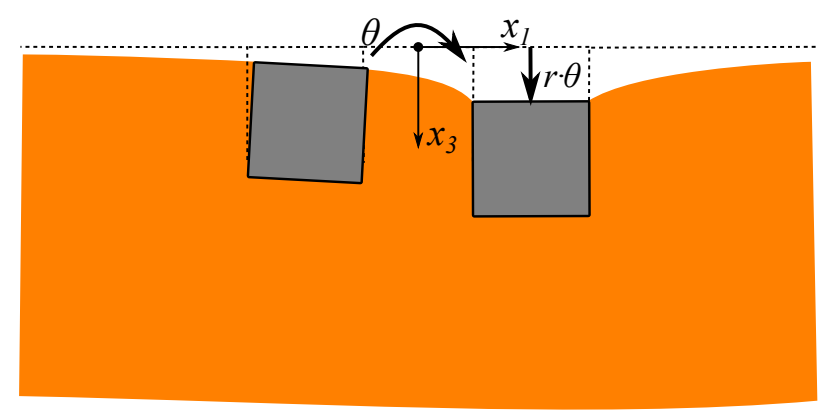

(a) Both foundations on different rotation sides

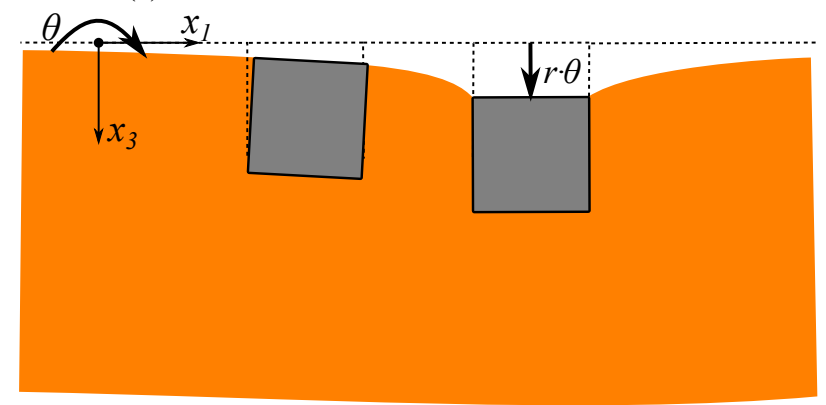

(b) Both foundations on the same rotation side

Figure 11: Illustration of the effect of a foundation vertical movement due to a rotation about an external axis (right) over another free-standing foundation (left) 

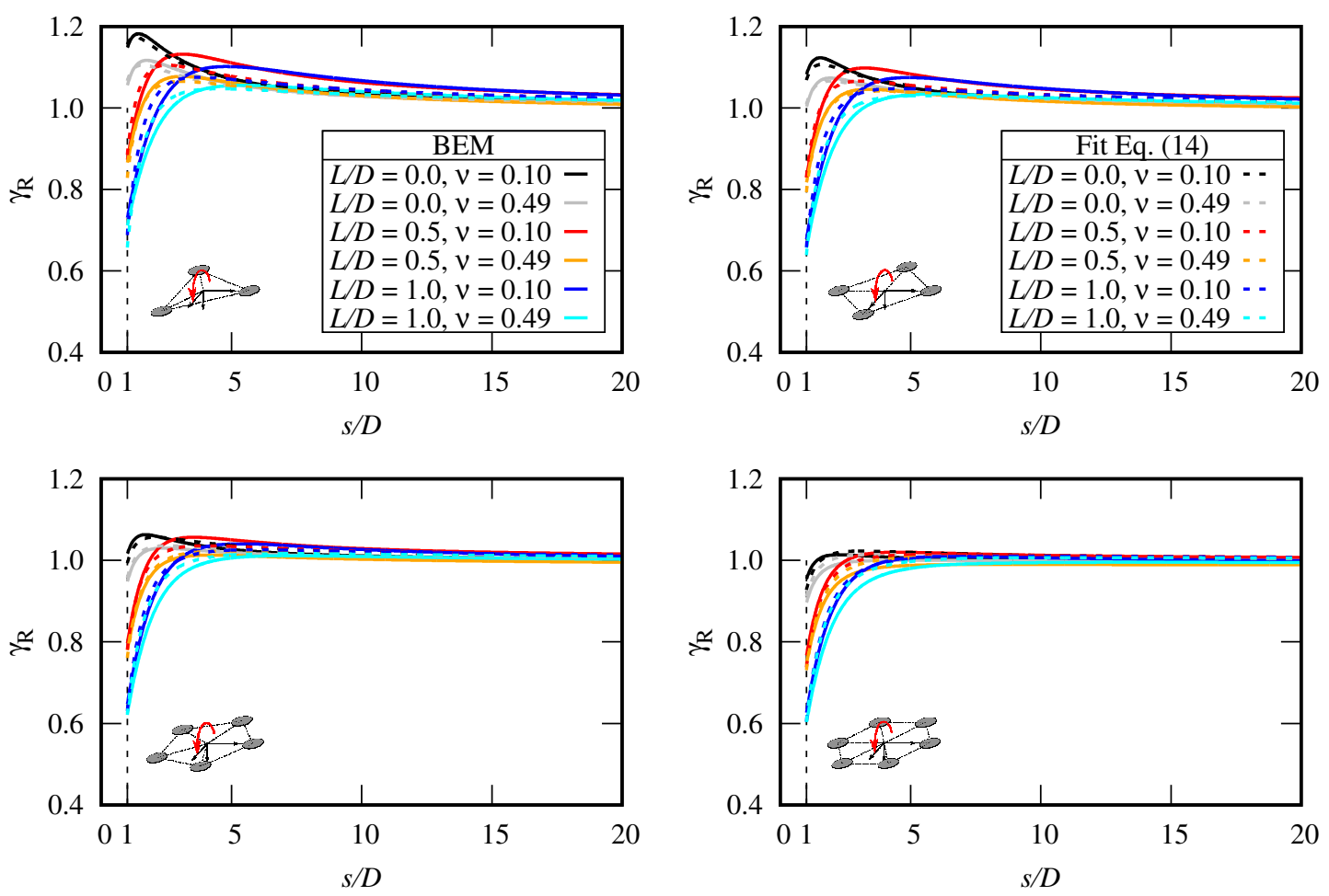

Figure 12: Correction factor $\gamma_{\mathrm{R}}=K_{\mathrm{R}}^{\mathrm{gr}} / K_{\mathrm{R}}^{\mathrm{grs}}$ for the rocking stiffness

We thus start by recalling that the vertical displacement due to an horizontal load $P$ acting in the $x$ direction and applied at $\left(x_{l}, y_{l}, z_{l}\right)=(0,0, c)$ is:

$$
w=\frac{P x}{16 \pi G(1-v)}\left[\frac{z-c}{R_{1}^{3}}+\frac{(3-4 v)(z-c)}{R_{2}^{3}}-\frac{6 c z(z+c)}{R_{2}^{5}}+\frac{4(1-v)(1-2 v)}{R_{2}\left(R_{2}+z+c\right)}\right]
$$

where $R_{1}=\sqrt{x^{2}+y^{2}+(z-c)^{2}}$ and $R_{2}=\sqrt{x^{2}+y^{2}+(z+c)^{2}}$, and the conventional notation $\left(x \equiv x_{1}, y \equiv x_{2}\right.$, $\left.z \equiv x_{3}\right)$ is here used instead of the indicial notation for the sake of clarity. Assuming that load and observation points are at the same vertical coordinate $L_{l}=z=c$, and considering a cylindrical coordinate system centered at the center of the regular polygon (see Fig. 4), the vertical displacement $w_{i j}$ of a point $j$ (foundation $j$ ) due to an horizontal load $P$ applied at a point $i$ (foundation $i$ ) is given by:

$$
w_{i j}=\frac{P x_{i j}}{16 \pi G(1-v)}\left[-\frac{12 L_{l}^{3}}{\left(x_{i j}^{2}+y_{i j}^{2}+4 L_{l}\right)^{5 / 2}}+\frac{4(1-v)(1-2 v)}{\left(x_{i j}^{2}+y_{i j}^{2}+4 L_{l}\right)+2 L_{l}\left(x_{i j}^{2}+y_{i j}^{2}+4 L_{l}\right)^{1 / 2}}\right]
$$

where $x_{i j}=r[\cos (2 \pi(j-1) / N)-\cos (2 \pi(i-1) / N)]$ and $y_{i j}=r[\sin (2 \pi(j-1) / N)-\sin (2 \pi(i-1) / N)]$. For $r \rightarrow$ $\infty$, we can assume that vertical and horizontal load-displacement relationships are those of isolated foundations (Appendix A]. Therefore, if we consider $P=K_{\mathrm{H}}^{\mathrm{f}}$, then $w_{i j}$ is the vertical displacement at $j$ due to anit horizontal displacement at $i$. Furthermore, if we multiply this $w_{i j}$ by $K_{\mathrm{V}}^{\mathrm{f}}$, then we obtain the resulting vertical force $f_{i j}=K_{\mathrm{V}}^{\mathrm{f}} w_{i j}$ at $j$ (for a fixed foundation at $j$ ) due to a unit displacement at $i$. The resulting moment with respect to the rotation axis is simply $m_{i j}=f_{i j} r \cos (2 \pi(j-1) / N)$. Taking the limit of $m_{i j}$ as $r \rightarrow \infty$ gives:

$$
m_{i j}^{\infty}=\lim _{r \rightarrow \infty} m_{i j}=\frac{(1-2 v) K_{\mathrm{V}}^{\mathrm{f}} K_{\mathrm{H}}^{\mathrm{f}}}{4 \pi G} \frac{r x_{i j}}{x_{i j}^{2}+y_{i j}^{2}} \cos (2 \pi(j-1) / N)
$$

where it must be noticed that all $r$ from the fraction $r x_{i j} /\left(x_{i j}^{2}+y_{i j}^{2}\right)$ cancel out, and only trigonometric functions remains. Moreover, $L_{l}$ also vanish, showing that Cerruti's solution lead to the same result. The total additional sway-rocking stiffness can be obtained by superposition. The summation of moments $m_{i j}$ for all loads $i=1, N$ and all observation points $j=1, N$ except when $i=j$ :

$$
K_{\mathrm{SR}}^{\mathrm{gra}}=\sum_{i=1}^{N} \sum_{j=1, j \neq i}^{N} m_{i j}^{\infty}
$$



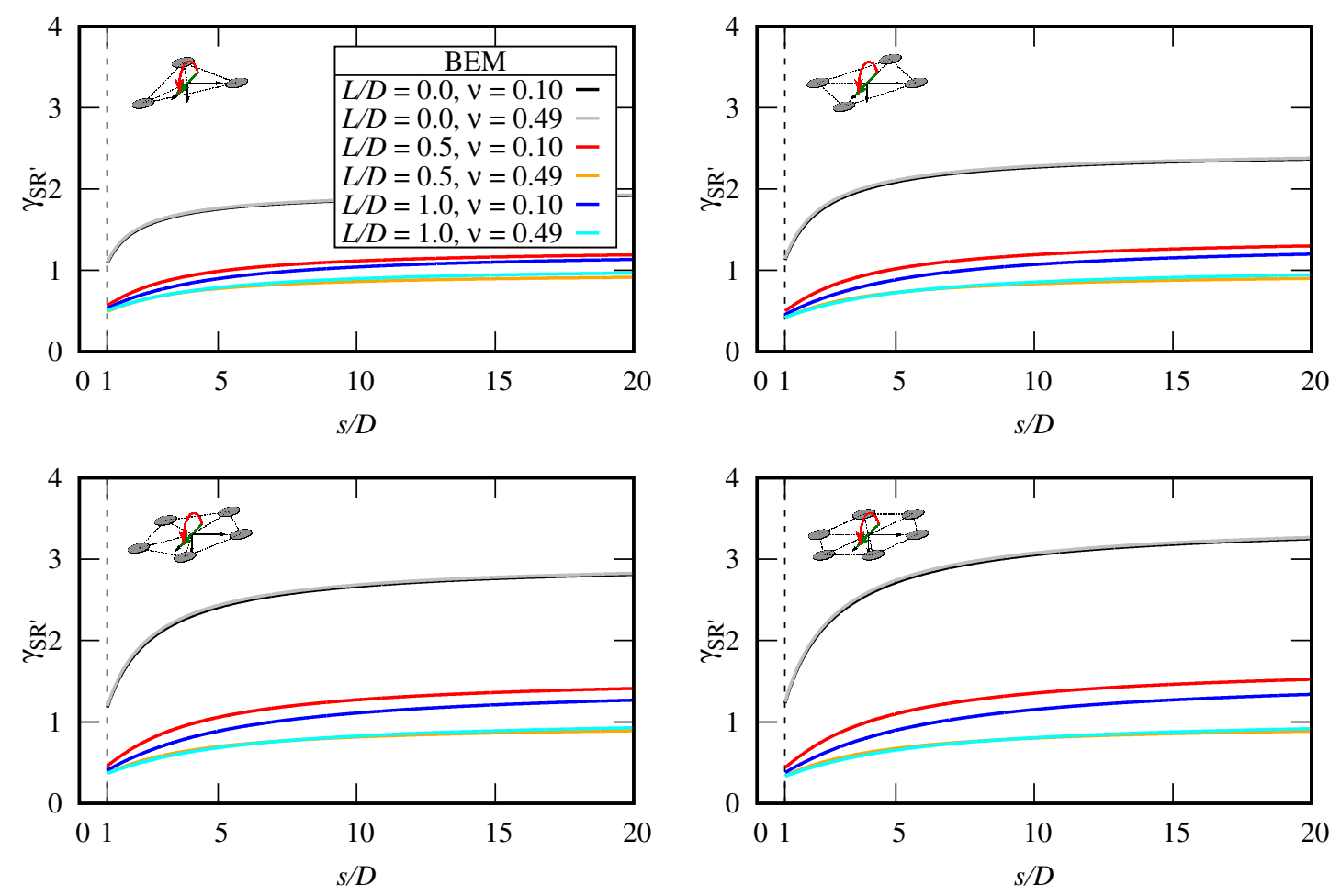

Figure 13: Correction factor $\gamma_{\mathrm{SR}^{\prime}}=K_{\mathrm{SR}}^{\mathrm{gr}} / K_{\mathrm{SR}}^{\mathrm{grs}}$ for the sway-rocking stiffness

This expression has been solved for a number of values of $N \geq 3$ using a computer algebra system, which, by induction, allow us to propose the following solution for arbitrary $N$ :

$$
K_{\mathrm{SR}}^{\mathrm{gra}}=N(N-1) \frac{(1-2 v) K_{\mathrm{V}}^{\mathrm{f}} K_{\mathrm{H}}^{\mathrm{f}}}{16 \pi G}
$$

The validity of this expression is shown in Fig. 14, where it can be seen that the correction factor using $K_{\mathrm{SR}}^{\mathrm{grs}}+K_{\mathrm{SR}}^{\mathrm{gra}}$ as the reference stiffness now tends to unity as $s / D \rightarrow \infty$. The group effect related to the proximity between foundations now follow a similar pattern to horizontal and vertical correction factors.

Given that the resulting correction factor is similar to the horizontal and vertical cases, it is assumed a similar type of function, but where the Poisson's ratio is now included given its influence. The following simple formula has been obtained via curve fitting of the results:

$$
\gamma_{\mathrm{SR}}=\frac{K_{\mathrm{SR}}^{\mathrm{gr}}}{K_{\mathrm{SR}}^{\mathrm{grs}}+K_{\mathrm{SR}}^{\mathrm{gra}}} \approx \frac{1}{1+2.27(1-2.06 / N)\left(1+1.39(1-0.96 v)(L / D)^{0.48}\right) /(s / D)}
$$

The average relative error of this formula is $3 \%$, while the maximum relative error reached is $15 \%$. The goodness of the proposed formula is shown in Fig. 14 using dashed lines.

\subsection{Torsional stiffness}

The group effect under static torsional loading of a group of foundations is in some ways similar to rocking loading but also to horizontal loading. By using the two foundation example, see Fig. 15, the torsional rotation of a foundation about its own axis produces a rotation in the opposite direction of the other free-standing foundation. On the other hand, the horizontal displacement of one of the foundations due to a torsional rotation with respect to the center of the polygon produces counteracting effects on the other foundation if located on the opposite side, but helping effects on other foundation if located on the same side.

Fig. 16) shows the obtained correction factor $\gamma_{\mathrm{T}}=K_{\mathrm{T}}^{\mathrm{gr}} / K_{\mathrm{T}}^{\text {grs }}$ for the torsional stiffness using solid lines, where $K_{\mathrm{T}}^{\mathrm{gr}}$ is obtained using the BEM model and $K_{\mathrm{T}}^{\mathrm{grs}}$ is obtained from (Eq. $(8)$ ). The first aspect to observe is the fact that, although in all cases the correction factor naturally tends to unity as $s / D \rightarrow \infty$, it does from values above or below unity depending on $N$ and $v$. Moreover, the tripod and tetrapod cases show a behavior very similar to rocking loading, while the pentapod and hexapod cases show a behavior similar to horizontal loading. This is explained by the fact that as $N$ increases, the interior angle of the polygon tends to $180^{\circ}$, i.e. torsion tends to produce coherent 

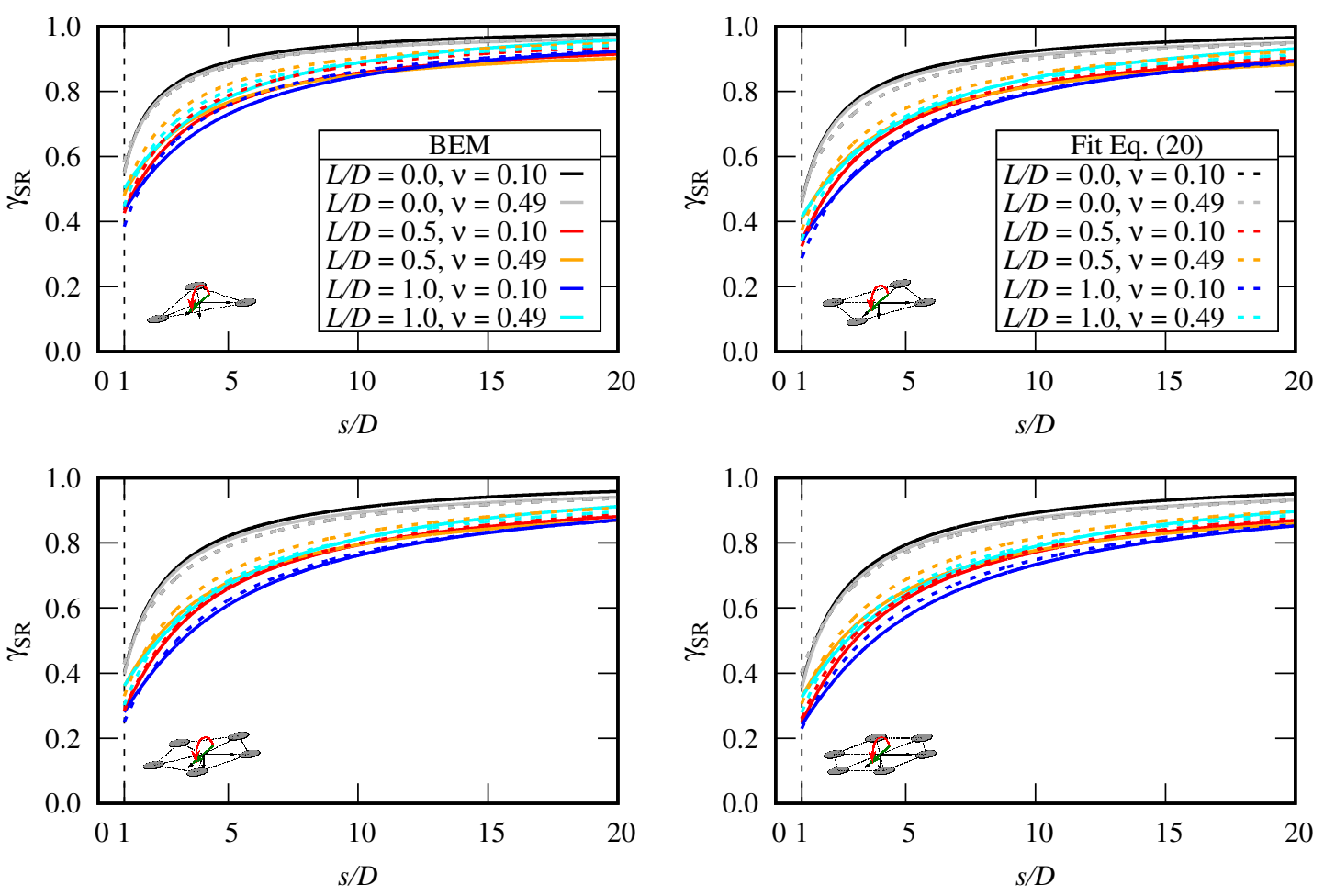

Figure 14: Correction factor $\gamma_{\mathrm{SR}}=K_{\mathrm{SR}}^{\mathrm{gr}} /\left(K_{\mathrm{SR}}^{\mathrm{grs}}+K_{\mathrm{SR}}^{\mathrm{gra}}\right)$ for the sway-rocking stiffness

displacements on neighboring foundations, and the helping effect similar to the horizontal loading case dominates. On the contrary, for small $N$ the counteracting effect of the torsional rotation dominates, showing a correction factor very similar to the rocking loading.

Unlike in the previous cases, no closed-form formula with the same level of simplicity and accuracy has been found for this correction factor.

\section{Relevance of group effects on fundamental frequency of OWTs}

In this section, the relevance of using group effects on the calculation of the first natural frequency of offshore wind turbines is studied. To this end, three multi-megawatt turbines are considered: Siemens SWT-3.6-107 [35], NREL 5-MW Reference Wind Turbine [36] and DTU 10-MW Reference Wind Turbine [37]. Table 1]collects the properties of these turbines relevant to this work.

\begin{tabular}{lccccc} 
Model & $P(\mathrm{MW})$ & $M_{\text {RNA }}(\mathrm{t})$ & $L_{\mathrm{t}}(\mathrm{m})$ & $D_{\mathrm{t}}(\mathrm{m})$ & $t_{\mathrm{t}}(\mathrm{mm})$ \\
\hline Siemens SWT-3.6-107 & 3.6 & 220 & 80 & 3.25 & 32.5 \\
NREL 5-MW Ref. WT & 5 & 350 & 90 & 4.9 & 23.5 \\
DTU 10-MW Ref. WT & 10 & 675 & 119 & 6.9 & 29
\end{tabular}

Table 1: Definition of OWTs used in the present study

Fig. 17 shows the 6-DOF model used to study the present problem, which consists of three nodes with horizontal displacement and rotation. At the hub level, a lumped mass is modeling the RNA $\left(M_{\mathrm{RNA}}\right)$. Both tower and substructure are Euler-Bernoulli beams made of steel $\left(E_{\mathrm{st}}=210 \mathrm{MPa}, \rho_{\mathrm{st}}=8000 \mathrm{~kg} / \mathrm{m}^{3}, v_{\mathrm{st}}=0.3\right)$, where a hollow cross section, with constant diameter $D_{\mathrm{t}}$ and thickness $t_{\mathrm{t}}$, is assumed for the tower, and the structural properties of the substructure are characterized as relative to those of the tower as $E I_{\mathrm{s}}=r_{\mathrm{I}} E I_{\mathrm{t}}$ and $A_{\mathrm{s}}=r_{\mathrm{A}} A_{\mathrm{t}}$, where $A$ and $E I$ are the cross-sectional area and inertia. The length of the tower is $L_{\mathrm{t}}$, and a variable substructure length $L_{\mathrm{s}}$ is considered. Added mass due to the fluid-substructure interaction can be safely neglected, as demonstrated by Moll et al. [38] for jackets. Different shear moduli $G$ and Poisson's ratios $v$ representing soft soils will be considered. Multi-bucket foundations with $N=3$ (tripod), $N=4$ (tetrapod), $N=5$ (pentapod) and $N=6$ (hexapod), with different center-to-center spacings $s$, foundation diameters $D$ and lengths $L$ are studied. The soil-foundation interaction is modeled with the proposed (corrected or uncorrected) static stiffness matrix. A modal analysis of this simple OWT model is used to obtain the first natural frequency. 


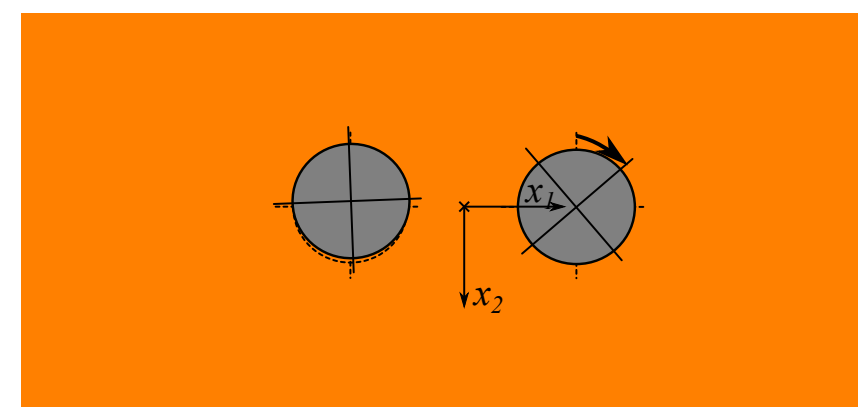

Figure 15: Illustration of the effect of a foundation torsional movement (right) over another free-standing foundation (left)
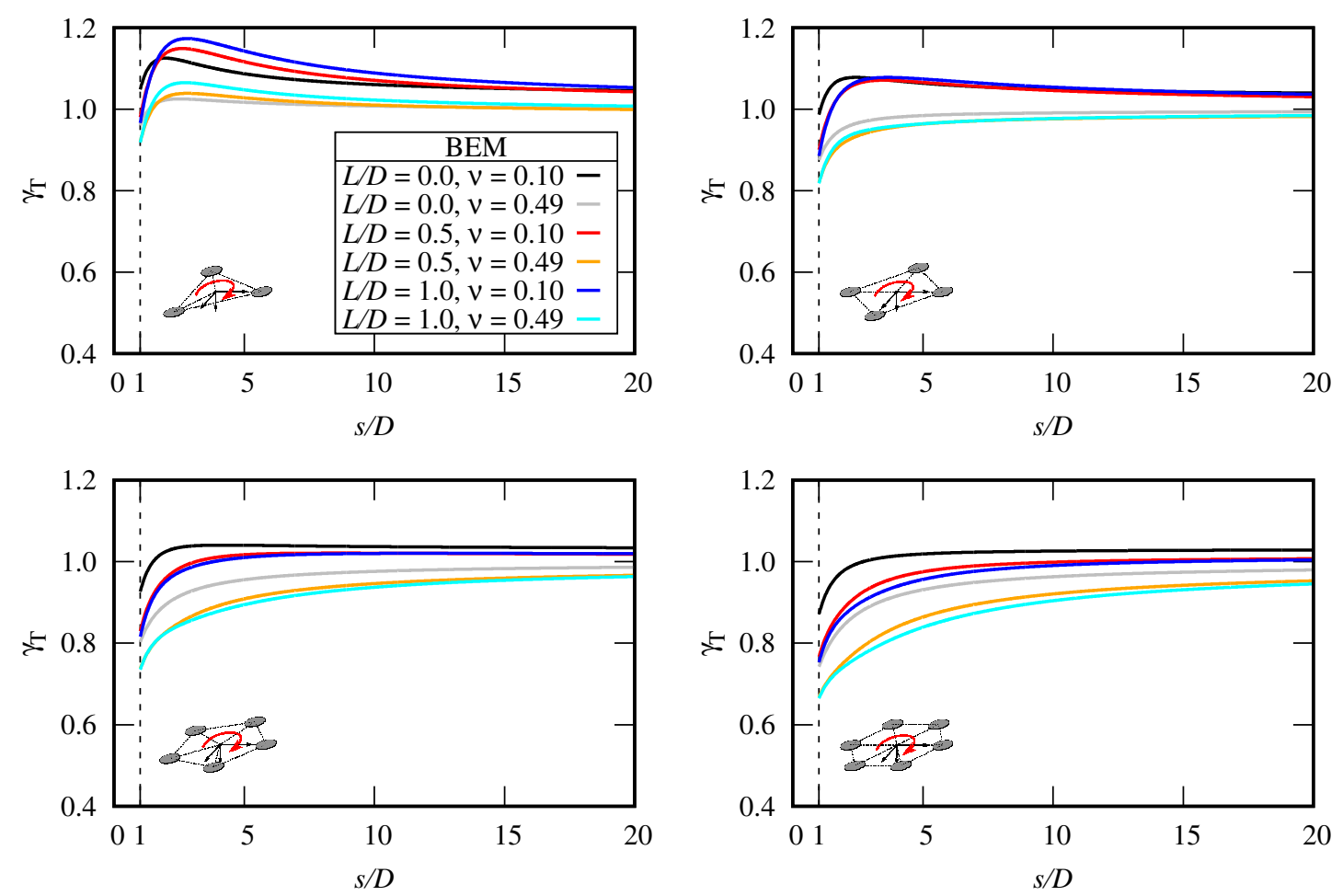

Figure 16: Correction factor $\gamma_{\mathrm{T}}=K_{\mathrm{T}}^{\mathrm{gr}} / K_{\mathrm{T}}^{\mathrm{grs}}$ for the torsional stiffness 


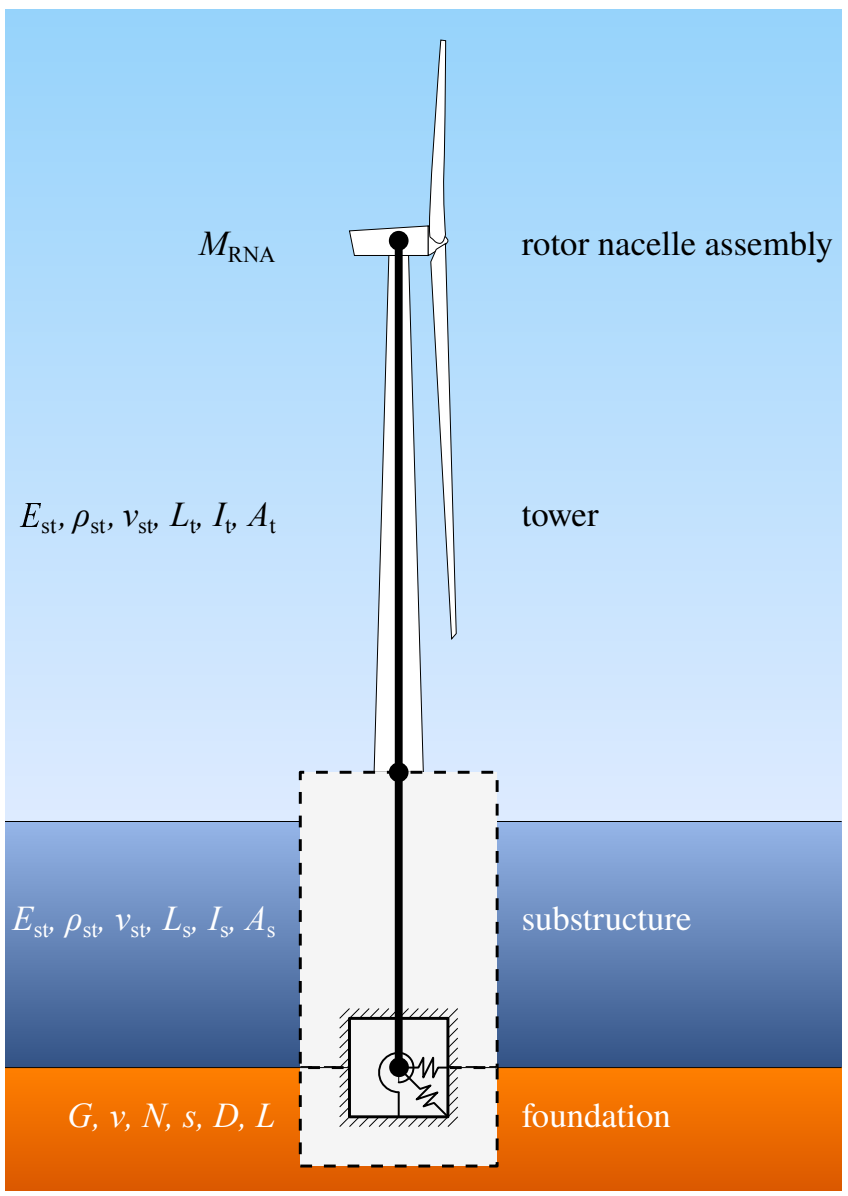

Figure 17: OWT model including soil-structure interaction 
The relevance of including or neglecting the group effects in the multi-bucket foundation will be assessed by presenting the ratio $f_{n}^{\prime} / f_{n}$ for different configurations, being $f_{n}$ the OWT fundamental frequency computed considering the simplified foundation stiffness matrix presented in Section 4 and $f_{n}^{\prime}$ the OWT fundamental frequency computed considering the corrected stiffnesses (computed through the closed-form correction factors provided in Section 5) where the interaction between elements is taken into account. It is worth mentioning here that the applicability of the closed-form correction factors to this analysis has been validated by comparison against the same functions computed using the stiffness functions obtained from the more rigorous BEM methodology presented in Section 3 (not shown for the sake of brevity), having found that the results are very close to each other within the margins provided in Section 5, and that both methodologies allow to draw the same conclusions.

Thus, Fig. 18 presents the ratio $f_{n}^{\prime} / f_{n}$ as a function of the bucket separation ratio $s / D$ for the Siemens SWT-3.6107 OWT mentioned above and for different configurations of the OWT system. Each subplot illustrates how the change in one of the system parameters (foundation shape ratio (a); bucket diameter (b); number of elements (c); substructure length (d), cross-section inertia (e) and area (f); soil Poisson's ratio (g); and soil stiffness (h)) affects the evolution of that ratio. In all those subplots, the variation of these parameters is studied around a starting base case, defined by the following values: $L / D=0.5, D=2 \mathrm{~m}, N=3, L_{\mathrm{s}}=30 \mathrm{~m}, r_{\mathrm{I}}=1, r_{\mathrm{A}}=1, v=0.49$ and $G=5 \mathrm{MPa}$.

In general, $f_{n}^{\prime} / f_{n}$ functions are non-monotonous with $s / D$, with a maximum located at $1.3<s / D<2.2$, a minimum value for $s / D=1$ (except in the case of surface footings, as seen in $L / D=0$ case) and, as could not be otherwise, a tendency to unity for largely spaced foundations. This means that, as expected, the more significant influence of the interaction between foundation elements arise when they are arranged very close to each other. However, the large variation of the $f_{n}^{\prime} / f_{n}$ functions for small separation ratios is very interesting because neglecting group effects leads to significant overestimations of the fundamental frequency $\left(f_{n}^{\prime} / f_{n}<1\right)$ in the case of extremely (unrealistic in some cases) close elements while, on the contrary, for slightly more spaced elements $(1.3<s / D<2.2$ depending on the specific configuration) the simplified model yields a significant underestimation of the system first natural frequency. In both cases, the error in the estimation of the OWT fundamental frequency due to neglecting the interaction between foundation elements can reach up to 5\% in any of the two directions.

Bucket shape ratio, bucket diameter and number of buckets are the system parameters that exert the largest influence on the computed fundamental frequencies. For $L / D=1$, the fundamental frequency is overestimated by the most simplified model if $s / D<1.5$, but tends to be underestimated for all other cases, especially for very shallow foundations, with the limit case of the surface footings yielding the maximum $f_{n}^{\prime} / f_{n}$ values. At the same time, the largest the bucket diameter, the less important the influence of the group effects, in such a way that for diameters $D \geq 4$, their influence can be considered negligible. On the other hand, the number of elements defines very clear tendencies. For multi-bucket fondations with more than four elements, the fundamental frequency is always overestimated when neglecting group effects, though the influence is negligible for $s / D \geq 2$, while in the case of tripods and tetrapods, the fundamental frequency tends to be underestimated except for really close cases. Here, it is worth highlighting that the largest influences appear for small groups $(N=3)$ or very large groups $(N=6$ or larger).

As for the rest of parameters, substructure length and mass (subplots (d) and (f)) present no significant influence on the ratio under study. Similarly, laterally stiffer substructures do not alter the conclusions drawn before in terms of fundamental frequency ratios (even tough this parameters affect the value of the fundamental frequencies themselves). Soil parameters, on the other hand, present a more significant influence. Soils with low Poisson's ratios (that could be used to represent unsaturated sands) yield a largest influence of the group effects when compared to soils with large Poisson's ratio (that could be used to represent saturated soils). At the same time, the influence of the group effects is more relevant in softer soils, for which the fundamental frequencies tend to be significantly underestimated in very soft soils.

In order to find out whether the size and inertia of the wind turbine may alter the conclusions drawn in the previous paragraphs, Fig. 19 presents the evolution of $f_{n}^{\prime} / f_{n}$ as a function of bucket separation ratio $s / D$ for the limit configurations presented in the previous figure but comparing the influence of the interaction between foundation elements over the NREL 5-MW (green lines) and the DTU 10-MW reference turbines (red lines) besides the previously studied Siemens SWT-3.6-107 turbine (black lines). The turbine itself does not alter the tendencies and main conclusions described above. The magnitude of the influence of considering the group effects increases with the size of the turbine, with maxima growing from approximately 3 to $5 \%$ from the smallest to the largest turbine under consideration.

\section{Conclusions}

In the present paper, the influence of the group effects on the stiffnesses of polygonally-arranged multi-bucket foundations for offshore wind turbines has been explored. To this end, a rigorous elastic boundary element model 

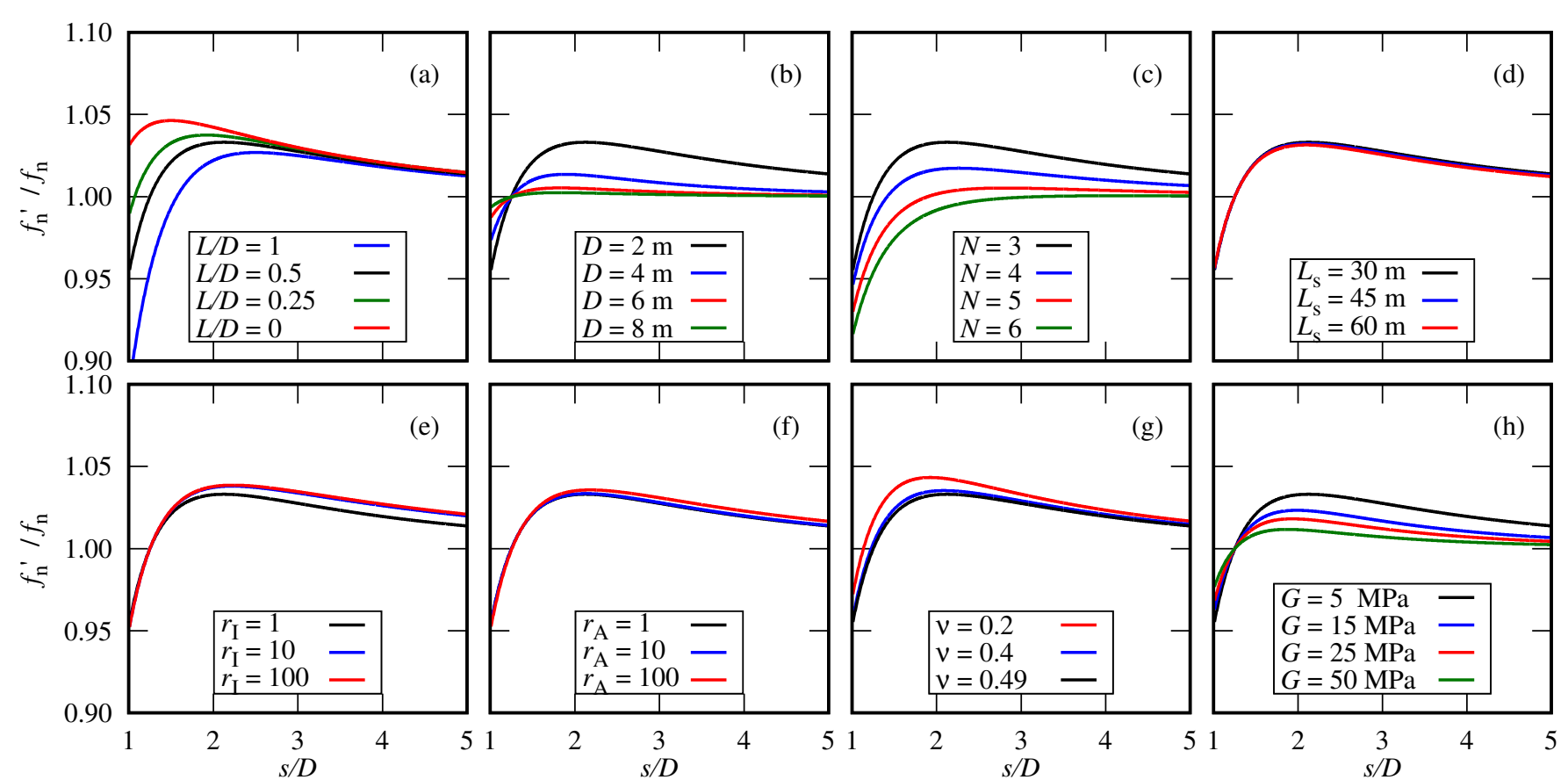

Figure 18: Ratio between OWT fundamental frequencies computed by including $\left(f_{\mathrm{n}}^{\prime}\right)$ or neglecting $\left(f_{\mathrm{n}}\right)$ foundation group effects. Siemens SWT-3.6-107. The base case (black solid line) corresponds to the following case: $L / D=$ $0.5, D=2 \mathrm{~m}, N=3, L_{\mathrm{s}}=30 \mathrm{~m}, r_{\mathrm{I}}=1, r_{\mathrm{A}}=1, v=0.49$ and $G=5 \mathrm{MPa}$.
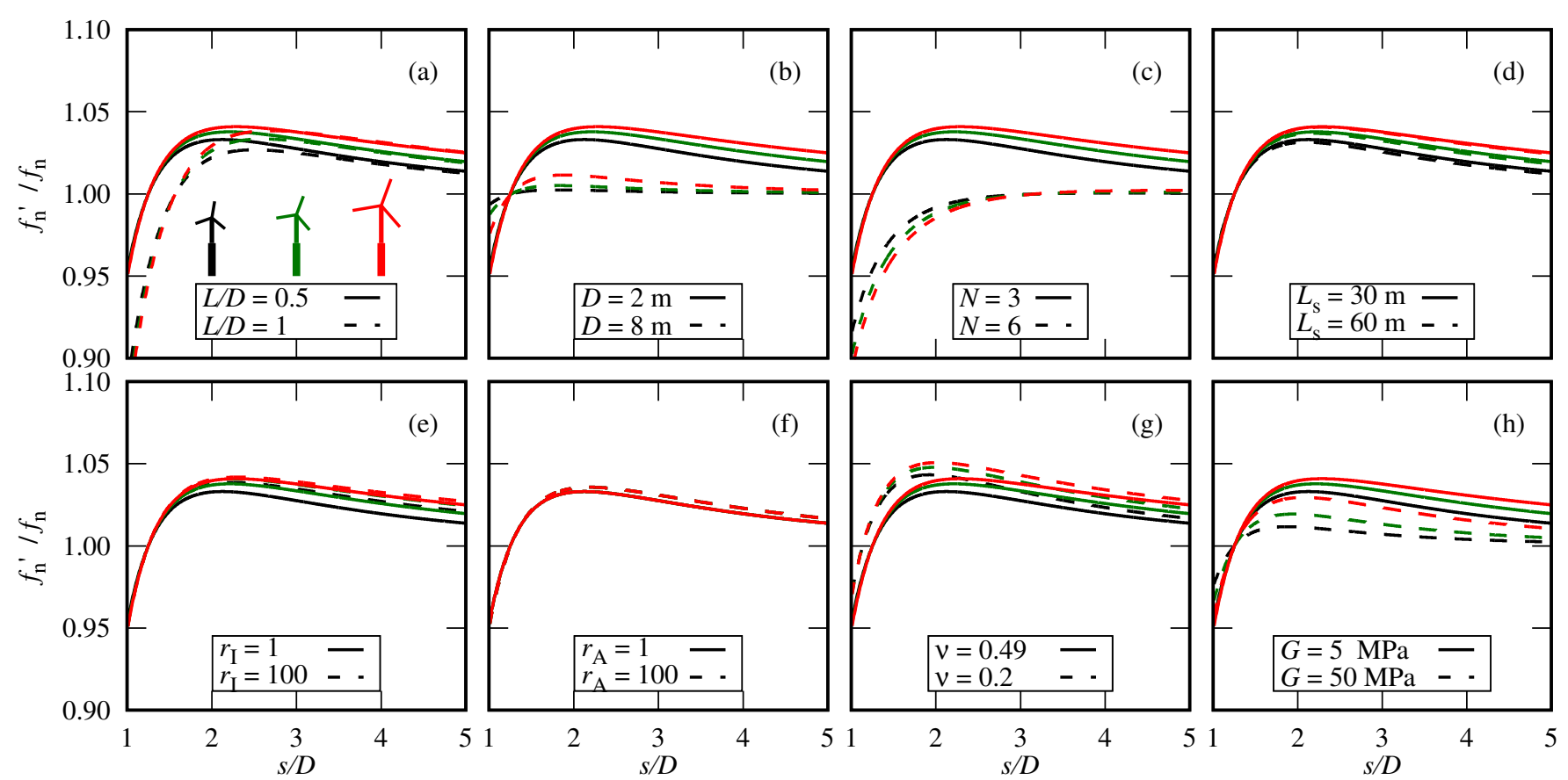

Figure 19: Ratio between OWT fundamental frequencies computed by including $\left(f_{\mathrm{n}}^{\prime}\right)$ or neglecting $\left(f_{\mathrm{n}}\right)$ foundation group effects. Siemens SWT-3.6-107 (black lines), NREL 5-MW (green lines) and the DTU 10-MW (red lines). The base case (black solid line) corresponds to the following case: $L / D=0.5, D=2 \mathrm{~m}, N=3, L_{\mathrm{S}}=30 \mathrm{~m}, r_{\mathrm{I}}=1$, $r_{\mathrm{A}}=1, v=0.49$ and $G=5 \mathrm{MPa}$. 
has been used to compute a set of correction factors representing the magnitude of the group effect. Such factors were later fitted into closed-form formulas that can be easily used to incorporate group effects to the common stiffness matrices obtained from the stiffnesses of the individual elements. In turn, these corrected stiffness functions can be used to assess SLS, FLS and target natural frequency requirements for OWT.

For translational stiffnesses (vertical and horizontal), correction factors are monotonous curves starting from around 0.5 when foundations are together, and smoothly approaching unity as foundation spacing gets large. They mainly depend on the spacing $s / D$, the foundation shape ratio $L / D$, and the number of foundations in the polygon $N$. At moderate spacings, say $s / D=3$, group stiffnesses are reduced to about $70 \%$.

For rotational stiffnesses (rocking and torsion), correction factors start from around 0.65 when foundations are together, then, depending on the foundation shape ratio $L / D$, number of foundations $N$ and Poisson's ratio $v$, they show a peak with magnitude greater than one, and finally they smoothly approach unity from above or below unity depending on the case as foundation spacing increases. These peaks are located between $s / D=1$ and $s / D=4$, and they can reach values up to 1.2 , although they reduce their values with the embedment and the number of foundations.

For the coupled sway-rocking stiffness, there exists an additional stiffness term to be added to those obtained from isolated foundations. Once this additional stiffness is added, the correction factor has a behavior similar to that of translational stiffnesses.

The influence, on the resulting fundamental frequencies of OWTs, of using foundation stiffnesses that include or neglect group effects is also tackled at the end of the paper. To do this, the alterations in the fundamental frequencies of a large set of systems, including different large turbines, foundation configurations, substructure configurations and soil properties, were presented. The fundamental frequency obtained without taking into account group effect may be misestimated up to $5 \%$ for closely-spaced foundations in soft soils. Besides, there exists large variations of the ratio between fundamental frequencies computed considering or not group effects, as that ratio can change very rapidly from $5 \%$ of overestimation to $5 \%$ in underestimation for different separation ratios. This highlights the importance of adequately modeling the interaction between foundation elements in these cases and, given that fundamental frequencies must be at least $10 \%$ away from operational $1 \mathrm{P}$ and $2 \mathrm{P} / 3 \mathrm{P}$ frequencies, it can be concluded that the group effect should not be neglected when computing the fundamental frequencies of OWTs founded on closely-spaced multi-bucket foundations in soft-soils.

The present findings establish the core for future work where the influence of the bucket specific geometry and flexibility [12] can be incorporated. The consideration of non-linear phenomena lies beyond the scope of the present work.

\section{Acknowledgements}

J.D.R. Bordón was recipient of the research fellowship FPU13/01224 and research short stay grant EST15/00521, both from the Ministerio de Educación, Cultura y Deportes of Spain. The authors are grateful for the support from the Ministerio de Economía y Competitividad (MINECO) of Spain, the Agencia Estatal de Investigación (AEI) of Spain and FEDER through Research Projects BIA2014-57640-R and BIA2017-88770-R. 


\section{A Stiffnesses of rigid cylindrical foundations}

Stiffnesses for a rigid cylindrical foundation completely bonded with the surrounding homogeneous soil $(0 \leq$ $L / D \leq 1,0 \leq v<0.5$ ) can be approximated as:

$$
\begin{aligned}
K_{\mathrm{V}}^{\mathrm{f}} & =\frac{2 G D \ln (3-4 v)}{1-2 v}\left[1+1.12(1-0.84 v)\left(\frac{L}{D}\right)^{0.84}\right] \\
K_{\mathrm{H}}^{\mathrm{f}} & =\frac{4 G D}{2-v}\left[1+1.83\left(\frac{L}{D}\right)^{0.74}\right] \\
K_{\mathrm{SR}}^{\mathrm{f}} & =\frac{11 G D^{2}}{4(15-17 v)}\left[1-2 v+20.7(1-v)\left(\frac{L}{D}\right)^{1.28}\right] \\
K_{\mathrm{R}}^{\mathrm{f}} & =\frac{G D^{3}}{3(1-v)}\left[1+(7.5-9 v)\left(\frac{L}{D}\right)+(10.5-7.7 v)\left(\frac{L}{D}\right)^{2.5}\right] \\
K_{\mathrm{T}}^{\mathrm{f}} & =\frac{2 G D^{3}}{3}\left[1+5.18\left(\frac{L}{D}\right)^{0.93}\right]
\end{aligned}
$$

where average relative errors after fitting the parameters with respect to BEM results are respectively: $0.8 \%$ (max. $1.9 \%), 1.1 \%$ (max. $2.4 \%$ ), $2.3 \%$ ( $\max .6 .0 \%$ ), $0.6 \%$ (max. $4.9 \%$ ), and $1.1 \%$ (max. $4.2 \%$ ). These formulas are based on Gazetas' methodology [27], where each stiffness component $K(G, v, D, L)$ is built from the product of the surface footing stiffness $K_{\text {surface }}(G, v, D)$ and an embedment dimensionless factor $\kappa(v, L / D)$.

\section{References}

[1] S. Bhattacharya. Challenges in design of foundations for offshore wind turbines. Engineering and Technology Reference, pages 1-19, 2014.

[2] W. Wang, Z. Gao, X. Li, T. Moan, and B. Wang. Model test and numerical analysis of an offshore bottom fixed pentapod wind turbine under seismic loads. In ASME 2016 35th International Conference on Ocean, Offshore and Arctic Engineering, 2016.

[3] Offshore standard DNV-OS-J101: design of offshore wind turbine structures. Det Norske Veritas, May 2014.

[4] L. Arany, S. Bhattacharya, J. Macdonald, and S. J. Hogan. Design of monopiles for offshore wind turbines in 10 steps. Soil Dynamics and Earthquake Engineering, 92:126-152, 2017.

[5] S. Jalbi and S. Bhattacharya. Closed form solution for the first natural frequency of offshore wind turbine jackets supported on multiple foundations incorporating soil-structure interaction. Soil Dynamics and Earthquake Engineering, 113:593-613, 2018.

[6] M. Shadlou and S. Bhattacharya. Dynamic stiffness of monopiles supporting offshore wind turbine generators. Soil Dynamics and Earthquake Engineering, 88:15 - 32, 2016.

[7] S. Jalbi, M. Shadlou, and S. Bhattacharya. Impedance functions for rigid skirted caissons supporting offshore wind turbines. Ocean Engineering, 150:21 - 35, 2018.

[8] J. P. Doherty and A. J. Deeks. Elastic response of circular footings embedded in a non-homogeneous halfspace. Géotechnique, 53(8):703-714, 2003.

[9] J. P. Doherty, G. T. Houlsby, and A. J. Deeks. Stiffness of flexible caisson foundations embedded in nonhomogeneous elastic soil. Journal of Geotechnical and Geoenvironmental Engineering, 131(12):1498-1508, 2005.

[10] F.M. Gelagoti, P.P. Lekkakis, R.S. Kourkoulis, and G. Gazetas. Estimation of elastic and non-linear stiffness coefficients for suction caisson foundations, chapter Geotechnical Engineering for Infrastructure and Development, pages 943-948. 2015.

[11] J. J. Aznárez, O. Maeso, and J. Domínguez. BE analysis of bottom sediments in dynamic fluid-structure interaction problems. Engineering Analysis with Boundary Elements, 30:124-136, 2006. 
[12] J. D. R. Bordón, J. J. Aznárez, and O. Maeso. Dynamic model of open shell structures buried in poroelastic soils. Computational Mechanics, 60(2):269-288, 2017.

[13] R. D. Mindlin. Force at a point in the interior of a semi-infinite solid. Journal of Applied Physics, 7:195-202, 1936.

[14] V. Mantič. A new formula for the C-matrix in the Somigliana identity. Journal of Elasticity, 33:191-201, 1993.

[15] G. N. Bycroft. Forced vibrations of a rigid circular plate on a semi-infinite elastic space and on an elastic stratum. Philosophical Transactions of the Royal Society A, 248(948):327-368, 1956.

[16] C. M. Gerrard and W. J. Harrison. The analysis of a loaded half space comprised of anisotropic layers. CSIRO Division of Applied Geomechanics, 10:20-21, 1970.

[17] D. A. Spence. Self similar solutions to adhesive contact problems with incremental loading. Proceedings of the Royal Society A, 305(1480):55-80, 1968.

[18] H. G. Poulos and E. H. Davies. Elastic solution for soil and rock mechanics. John Wiley \& Sons, New York, 1974.

[19] H. Borowicka. Uber ausmittig belaste starre platten auf elastich-isotropem untergrund. Ingenieur-Archiv, $1: 1-8,1943$.

[20] E. Reissner and H. F. Sagoci. Forced torsional oscillations of an elastic half-space. I. Journal of Applied Physics, 15(652):652-654, 1944.

[21] E. Kausel. Forced vibrations of circular foundations on layered media. PhD thesis, Department of Civil Engineering - Massachusetts Institute of Technology, 1974.

[22] A. Pais and E. Kausel. Approximate formulas for dynamic stiffnesses of rigid foundations. Soil Dynamics and Earthquake Engineering, 7(4):213-227, 1988.

[23] R. Abascal. Estudio de problemas dinámicos en interacción suelo-estructura por el método de los elementos de contorno. PhD thesis, Escuela Técnica Superior de Ingenieros Industriales de la Universidad de Sevilla, 1984.

[24] J. Domínguez. Boundary Elements in Dynamics. International Series on Computational Engineering. Computational Mechanics Publications, 1993.

[25] J. P. Wolf. Dynamic soil-structure interaction. Civil Engineering and Engineering Mechanics. Prentice-Hall, Inc., 1985.

[26] J. P. Wolf. Soil-structure-interation analysis in time domain. Prentice-Hall, Inc., 1988.

[27] G. Gazetas. Formulas and charts for impedances of surface and embedded foundations. Journal of Geotechnical Engineering, 117(9):1363-1381, 1991.

[28] J. G. Sieffert. What is missing for using in practice available results on foundation impedances. In Eleventh World Conference on Earthquake Engineering, Paper No. 2075. Elsevier Science Ltd, 1996.

[29] R. D. Cook, D. S. Malkus, and M. E. Plesha. Concepts and applications of finite element analysis. John Wiley \& Sons, third edition, 1989.

[30] H. G. Poulos and E. H. Davies. Pile foundation analysis and design. Rainbow-Bridge Book Co., 1980.

[31] J. E. Bowles. Foundation analysis and design. McGraw-Hill, 1996.

[32] M. F. Randolph and C. P. Wroth. An analysis of the vertical deformation of pile groups. Géotechnique, 29(4):423-439, 1979.

[33] H. L. Wong and J. E. Luco. Dynamic interaction between rigid foundations in a layered half-space. Soil Dynamics and Earthquake Engineering, 5(3):149-158, 1986.

[34] V. Cerruti. Sulla deformazione di uno strato isotropo indefinito limitato da due piani paralleli. Atti Della Accademia Nazionale Dei Lincei, 1(4):521-522, 1884. 
[35] D. Lombardi. Dynamics of offshore wind turbines. Master's thesis, University of Bristol, 2010.

[36] J. Jonkman, S. Butterfield, W. Musial, and G. Scott. Definition of a 5-MW Reference Wind Turbine for Offshore System Development. Technical Report NREL/TP-500-38060, National Renewable Energy Laboratory, February 2009.

[37] C. Bak, F. Zahle, R. Bitsche, T. Kim, A. Yde, L. C. Henriksen, M. H. Hansen, J. Blasques, M. Gaunaa, and A. Natarajan. Description of the DTU 10-MW Reference Wind Turbine. Technical Report I-0092, DTU Wind Energy, July 2013.

[38] H. Moll, F. Vorpahl, and H. Busmann. Dynamic of support structures for offshore wind tturbine in fullycoupled simulations - influence of water added mass on jacket mode shapes, natural frequencies and loads. In Proceedings of the European Wind Energy Conference, volume 5, pages 3701-3707, Warsaw, Poland, 2010. EVEA. 